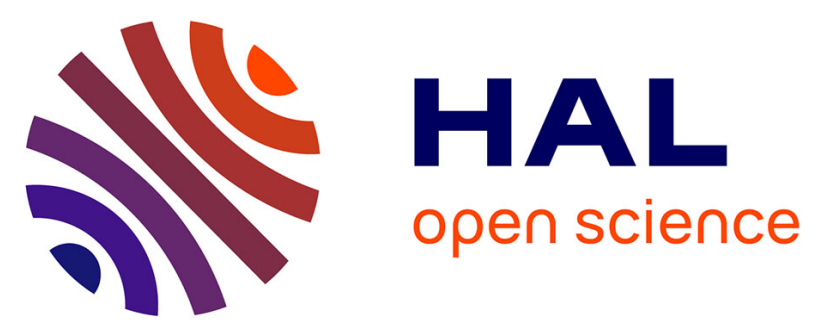

\title{
Simultaneous multi-slice T1 mapping using MOLLI with blipped CAIPIRINHA bSSFP
}

Zakarya Bentatou, Thomas Troalen, Monique Bernard, Maxime Guye, Lauriane Pini, Axel Bartoli, Alexis Jacquier, Stanislas Rapacchi, Frank Kober

\section{- To cite this version:}

Zakarya Bentatou, Thomas Troalen, Monique Bernard, Maxime Guye, Lauriane Pini, et al.. Simultaneous multi-slice T1 mapping using MOLLI with blipped CAIPIRINHA bSSFP. Magnetic Resonance Imaging, 2020, 10.1016/j.mri.2020.03.006 . hal-02547998

\section{HAL Id: hal-02547998 \\ https://hal-amu.archives-ouvertes.fr/hal-02547998}

Submitted on 13 May 2020

HAL is a multi-disciplinary open access archive for the deposit and dissemination of scientific research documents, whether they are published or not. The documents may come from teaching and research institutions in France or abroad, or from public or private research centers.
L'archive ouverte pluridisciplinaire HAL, est destinée au dépôt et à la diffusion de documents scientifiques de niveau recherche, publiés ou non, émanant des établissements d'enseignement et de recherche français ou étrangers, des laboratoires publics ou privés. 


\section{Simultaneous multi-slice T1 mapping using MOLLI with Blipped CAIPIRINHA bSSFP}

Zakarya Bentatou ${ }^{1,2,3}$, MSc; Thomas Troalen ${ }^{3}, \mathrm{PhD}$; Monique Bernard ${ }^{1}, \mathrm{PhD}$; Maxime

Guye $^{1,2}$, MD PhD; Lauriane Pini ${ }^{1,2}$, BSc; Axel Bartoli ${ }^{4}$, MD; Alexis Jacquier ${ }^{1,4}$, MD PhD;

Frank Kober ${ }^{1}, \mathrm{PhD}$; Stanislas Rapacchi ${ }^{1,2}, \mathrm{PhD}$

1. Aix Marseille Univ, CNRS, CRMBM, Marseille, France

2. APHM, Hôpital Universitaire Timone, CEMEREM, Marseille, France

3. Siemens Healthcare SAS, Saint-Denis, France

4. APHM, Hôpital Universitaire Timone, Service de Radiologie, Marseille, France

Zakarya Bentatou: zakarya.bentatou@etu.univ-amu.fr

Thomas Troalen: thomas.troalen@siemens-healthineers.com

Monique Bernard: monique.bernard@univ-amu.fr

Maxime Guye: maxime.guye@ap-hm.fr

Lauriane Pini: lauriane.pini@univ-amu.fr

Axel Bartoli: axel.bartoli@ap-hm.fr

Alexis Jacquier: alexis.jacquier@ap-hm.fr

Frank Kober: frank.kober@univ-amu.fr

Stanislas Rapacchi : stanislas.rapacchi@univ-amu.fr

Corresponding author:

Zakarya BENTATOU

bentatouzakaria@gmail.com

CEMEREM - TIMONE PAVILLON 3

CENTRE HOSPITALIER REGIONAL DE MARSEILLE - APHM

264 rue Saint-Pierre

13385 MARSEILLE CEDEX 05

Phone : +33491384807 


\section{Abstract \\ Background}

This study evaluates the possibility for replacing conventional 3 slices, 3 breath-holds MOLLI cardiac T1 mapping with single breath-hold 3 simultaneous multi-slice (SMS3) T1 mapping using blipped-CAIPIRINHA SMS-bSSFP MOLLI sequence. As a major drawback, SMSbSSFP presents unique artefacts arising from side-lobe slice excitations that are explained by imperfect RF modulation and bSSFP low flip angle enhancement. Amplitude-only RF modulation (AM) is proposed to reduce these artefacts in SMS-MOLLI compared to conventional Wong multi-band RF modulation (WM).

\section{Materials and methods}

Phantoms and ten healthy volunteers were imaged at $1.5 \mathrm{~T}$ using a modified blippedCAIPIRINHA SMS-bSSFP MOLLI sequence with 3 simultaneous slices. WM-SMS3 and AMSMS3 were compared to conventional single-slice (SMS1) MOLLI. First, SNR degradation and $\mathrm{T} 1$ accuracy were measured in phantoms. Second, artefacts from side-lobe excitations were evaluated in a phantom designed to reproduce fat presence near the heart. Third, the occurrence of these artefacts was observed in volunteers, and their impact on T1 quantification was compared between WM-SMS3 and AM-SMS3 with conventional MOLLI as a reference.

\section{Results}

In the phantom, larger slice gaps and slice thicknesses yielded higher SNR. There was no significant difference of T1 values between conventional MOLLI and SMS3-MOLLI (both WM and AM). Positive banding artefacts were identified from fat neighbouring the targeted FOV due to side-lobe excitations from WM and the unique bSSFP signal profile. AM RF pulses reduced these artefacts by $38 \%$.

In healthy volunteers, AM-SMS3-MOLLI showed similar artefact reduction compared to WMSMS3-MOLLI ( $3 \pm 2$ vs $5 \pm 3$ corrupted LV segments out of 16$)$. In-vivo native T1 values 
obtained from conventional MOLLI and AM-SMS3-MOLLI were equivalent in LV myocardium (SMS1-T1=935.5 $\pm 36.1 \mathrm{~ms} ;$ AM-SMS3-T1=933.8 $\pm 50.2 \mathrm{~ms} ; \mathrm{P}=0.436)$ and LV blood pool (SMS1-T1=1475.4 $\pm 35.9 \mathrm{~ms} ;$ AM-SMS3-T1=1452.5 $\pm 70.3 \mathrm{~ms} ; \mathrm{P}=0.515)$. Identically, no differences were found between SMS1 and SMS3 postcontrast T1 values in the myocardium $(\mathrm{SMS} 1-\mathrm{T} 1=556.0 \pm 19.7 \mathrm{~ms} ; \mathrm{SMS} 3-\mathrm{T} 1=521.3 \pm 28.1 \mathrm{~ms} ; \mathrm{P}=0.626)$ and the blood (SMS1-T1= 478 $\pm 65.1 \mathrm{~ms} ; \mathrm{AM}-\mathrm{SMS} 3-\mathrm{T} 1=447.8 \pm 81.5 ; \mathrm{P}=0.085)$.

\section{Conclusions}

Compared to WM RF modulation, AM SMS-bSSFP MOLLI was able to reduce side-lobe artefacts considerably, providing promising results to image the three levels of the heart in a single breath hold. However, few artefacts remained even using AM-SMS-bSSFP due to residual RF imperfections. The proposed blipped-CAIPIRINHA MOLLI T1 mapping sequence provides accurate in vivo $\mathrm{T} 1$ quantification in line with those obtained with a single slice acquisition.

\section{Keywords}

Simultaneous multi-slice, CAIPIRINHA, blipped-CAIPIRINHA, bSSFP, T1 mapping, MOLLI, amplitude-only modulation, Wong modulation. 


\section{List of abbreviations}

MOLLI: Modified Look-Locker Imaging

SMS: simultaneous multi-slice

bSSFP: balanced steady-state free precession

RF: radiofrequency

AM: amplitude-only modulation

WM: Wong modulation

SNR: signal-to-noise ratio

LV: left ventricle

CAIPIRINHA: Controlled Aliasing in Parallel Imaging Results in Higher Acceleration FOV: field of view

CMR: cardiac magnetic resonance

SPGR: spoiled gradient-echo readout

EPI: Echo planar imaging

FA: flip angle

ACS: auto-calibration signal

GRAPPA: Generalized Autocalibrating Partially Parallel Acquisition

BW: bandwidth

FLASH: Fast Low Angle Shot

TBW: time-bandwidth product

ROI: region of interest

SD: standard deviation 


\section{Introduction}

Myocardial T1 is now considered as an important biomarker for in vivo tissue characterization and quantification of fibrosis [1], [2]. The modification of native T1 values has been observed in a large range of cardiac diseases where fibrosis often plays an important role linked to Extracellular volume fraction changes [3]-[9], but T1 values can also be altered in the cases of amyloidosis [10], thalassemia [11] or blood volume changes [12].

Among the various techniques that have been proposed for cardiac T1 mapping, Modified Look-Locker Imaging [13] (MOLLI) is currently the most frequently used due to its high precision and reproducibility [14]. One of the challenges of cardiac MRI is breathing motion, which is dealt with by performing breath-holds during acquisitions. Using the MOLLI sequence with balanced steady-state free precession (bSSFP) readout, one slice can be acquired in 10 to 15 seconds under breath-hold [13]. But for patients with arrhythmia, even a breath-hold of 15 seconds can be hard to achieve and maintain when done several times within the same examination. Multiple techniques have been proposed to accelerate MOLLI [15]-[24], however none so far have considered acquiring multiple slices simultaneously.

Simultaneous multi-slice (SMS) imaging [25] techniques allow the reduction of acquisition time by exciting multiple slices simultaneously using multiband radiofrequency (RF) pulses. For T1 mapping acquisitions, SMS is an interesting solution allowing the acquisition of the three slices basal, median and apical in a single breath-hold.

Controlled Aliasing in Parallel Imaging Results in Higher Acceleration (CAIPIRINHA) [26] is a preferred technique for SMS acquisitions, because it allows increasing the distance between superimposed pixels from slices acquired simultaneously, which facilitates the unwrapping of images. This distance is increased by shifting the slices with respect to each other in the imaging FOV. Slice shifting corresponds to a linear phase increment within k-space, usually obtained by phase increments between adjacent k-space lines from different RF excitations or gradient 
blips. CAIPIRINHA has been applied to spoiled gradient-echo readout (SPGR) using RF phase cycling. In CMR, CAIPIRINHA-SPGR has been applied to cine [27] and cardiac T1 mapping with the Saturation pulse-prepared heart rate independent inversion recovery (SAPPHIRE) sequence [28]. However, bSSFP remains the primary choice for readout in CMR T1 mapping at $1.5 \mathrm{~T}$, due to its higher contrast and SNR.

CAIPIRINHA-SMS may show its potential for accelerating CMR if combined with bSSFP readout [29], [30]. But, concurrently performing CAIPIRINHA RF increments and maintaining the periodic RF cycling from the bSSFP sequence greatly limits the compatibility of the two and stands as a technical challenge.

SMS-accelerated bSSFP imaging was introduced by Stäb et al. [29], who defined a particular RF phase cycle that, for the simultaneous acquisition of 2 slices (SMS-2), provided a FOV/2 shift for the $2^{\text {nd }}$ slice (i.e. an alternate $\pi$ shift every other line). While one slice undergoes a RF phase cycle with $\pi / 2$ increments, the second slice experiments $-\pi / 2$ increment cycles (or $3 \pi / 2$ increments). The undesired consequences of these modified RF phase cycles are the corresponding shifts of the frequency response in off-resonance phase of $\pi / 2$ and $-\pi / 2$, respectively. Thus, CAIPIRINHA-induced displacements of the bSSFP banding artifacts are observed within slices and differ between each simultaneously acquired slice. To circumvent this issue, Stäb and Speier [30] recently proposed Gradient-controlled local Larmor adjustment (GC-LOLA) to unbalance the gradients along the slice encoding direction to shift back the frequency response profiles from all slices. An additional pair of gradients along the slice encoding direction compensates for the phase accrual induced by RF phase. One major drawback of this extra pair of gradients is the integration of the spins' frequency response across the slice thickness, which widens the stop bands and thus renders this SMS-bSSFP approach more demanding in terms of B0 homogeneity. 
An alternative approach to SMS-bSSFP is to maintain the bSSFP RF phase cycle intact and to rely on slice-gradient blips [31] resulting in a linear increment of the phase between slices prior to readout, at each TR. This approach draws from the blipped-CAIPIRINHA techniques employed in Echo Planar Imaging (EPI)[32]. To maintain balanced gradients at each new excitation, the exact negative slice-gradient blip is added after each readout (Figure 1). As a benefit from this approach, the bSSFP frequency response is preserved, and banding artefacts are not different from those in non-accelerated bSSFP. This technique, termed blipped CAIPIRINHA, was recently integrated in cine MRI [33] with promising results. In this study, blipped-CAIPIRINHA is evaluated for single-shot bSSFP in the MOLLI sequence.

To better handle power peaks occurring by RF superposition in the implementation of CAIPIRINHA, Wong et al. proposed a phase optimization of multiband pulses [34]. With this modulation scheme, however, side-lobe excitations were shown to produce signal from nontargeted slices [35]. Using bSSFP in particular, the occurrence of this signal led to unresolved positive banding in the context of cardiac imaging as shown by Landes et al. [36]. Indeed, imperfect rendering of multiband RF pulses can lead to excitation of side-lobes in sliceencoding direction. These side-lobes correspond to non-targeted slice excitations with very low flip angles. However, bSSFP has a unique frequency response at low flip angles with high signal magnitudes in proximity to off-resonance stop-bands (see Appendix A). Thus, positive banding signals from un-targeted slices can arise in SMS-bSSFP images and cannot be resolved by the SMS unaliasing reconstruction due to the missing correspondence in calibration data.

Recently, Abo Seada et al. [35] proposed a tailored multiband RF design using amplitude-only modulation that mitigates the imperfect hardware rendering of multiband RF pulses. As such, amplitude-modulated multiband RF pulses demonstrated reduction of side-lobe excitations in a spoiled gradient-echo sequence when compared to conventional multiband RF optimizations such as that proposed by Wong [34] . This tailored design uses a single dimension for RF 
modulation to avoid rapid bidimensional oscillations and simplify RF rendering when compared to conventionally real and imaginary Wong's multiband RF optimization.

In this study, the two approaches for RF design (amplitude-modulated and Wong's optimized modulation, in the following named AM and WM, respectively) were evaluated in the context of a single-shot blipped SMS-bSSFP MOLLI sequence. First, blipped-CAIPIRINHA SMSbSSFP (both AM and WM) was evaluated in a phantom to observe the influence of slice thickness, slice distance and pixel bandwidth on image SNR. Second, T1 quantification accuracy and precision using the proposed AM-SMS-MOLLI was assessed in calibrated T1 phantoms. Third, the relative intensity of side-lobe artefacts was compared between WM and AM designs for SMS-bSSFP. And fourth, T1 maps from SMS-MOLLI was compared to singleslice reference T1 maps from conventional MOLLI, in a sample of healthy volunteers using the two RF designs to appreciate the corresponding severity of side-lobe excitation artefacts in vivo. 


\section{Material and methods}

All CMR experiments were performed on a 1.5T Avanto Scanner (Siemens Healthineers, Erlangen, Germany) using a dedicated 32-channel cardiac coil array (InVivo, Siemens, Gainesville, Florida, USA). The in vivo study was approved by the local ethical board committee and conducted according to the modified Declaration of Helsinki. Ten healthy volunteers (7 men and 3 women) were recruited (COFLORES clinical trial NCT02848703, www.clinicaltrials.gov ) and enrolled for a cardiac MRI examination after providing informed consent. Images for both phantom and volunteers were acquired using a custom blippedCAIPIRINHA-bSSFP MOLLI, where gradient blips areas are standardized to obtain FOV/N shift for each slice where $\mathrm{N}$ is the number of SMS slices. The area varies for each TR, cycling every N TR (Figure 1) with the following parameters, unless stated otherwise: FOV $=341 \times 350 \mathrm{~mm}^{2}, \quad$ spatial resolution $=1.87 \times 1.87 \mathrm{~mm}^{2}, \quad$ TR $/ \mathrm{TE} / \mathrm{flip}$ angle $(\mathrm{FA})=$ $2.76 \mathrm{~ms} / 1.19 \mathrm{~ms} / 35^{\circ}$, pixel band width $(\mathrm{BW})=800 \mathrm{~Hz} / \mathrm{px}$, slice thickness $=8 \mathrm{~mm}$, slice gap $=20 \mathrm{~mm}$ and a Generalized Autocalibrating Partially Parallel Acquisition (GRAPPA) factor 2 with 48 external auto-calibration signal (ACS) lines. Sinc-shaped excitation pulse duration was set to 1ms. Breath-hold durations for in-vivo examinations were between 11 and 13s depending on the volunteers' heart rate.

\subsection{Phantom experiments}

\subsubsection{SNR evaluation}

The American College of Radiology (ACR) MRI phantom [37] has been used for this experiment. Twenty single-shot SMS3-bSSFP images were acquired at 1s intervals without inversion preparation using the following parameters: $F O V=320 \times 320 \mathrm{~mm}^{2}$, spatial resolution: $2 \times 2 \mathrm{~mm}^{2}, \mathrm{TR} / \mathrm{TE} / \mathrm{FA}=3.81 \mathrm{~ms} / 1.49 \mathrm{~ms} / 30^{\circ}, \mathrm{BW}=600,800,1000,1200$ and $1400 \mathrm{~Hz} / \mathrm{px}$ (For all parameters, please refer to Appendix B, Table 1). For SMS and GRAPPA reconstruction calibration, a set of 48 low-resolution k-space lines was acquired 1s prior to the accelerated 
acquisition using a FLASH readout $\left(64 \times 48\right.$ matrix, $5^{\circ}$ flip angle, TR/TE=4.0ms $\left./ 2.0 \mathrm{~ms}\right)$. In the first part of the experiment, the slice thickness was varied from 3 to $8 \mathrm{~mm}$ ( $1 \mathrm{~mm}$ step) and the gap between slices was fixed at $48 \mathrm{~mm}$. For the second part, slice thickness was fixed at $6 \mathrm{~mm}$, to consider a realistic scenario for CMR conditions with difficult conditions for SMS as small slice thickness produces more noisy SMS images, and the slice gap varied from 200 to $800 \%$ of the slice thickness with a $100 \%$ step. For both experiments, images were acquired without SMS and using WM-SMS3 and AM-SMS3, for comparison. The temporal variance was used to assess SNR. Hence, SNR was defined as the time series' mean intensity over intensity standard deviation. To remove signal acquired before reaching the steady state, the five first images were discarded from each time series.

\subsubsection{T1 quantification}

SMS1-T1 and SMS3-T1 maps were acquired on a calibrated relaxometry phantom with 12 tubes of Agarose gel (Eurospin II, Diagnostic Sonar LTD, Scotland) using conventional MOLLI and SMS accelerated MOLLI, respectively, with the recommended 5(3)3 scheme [1] and the acquisition parameters described above but with $\mathrm{FOV}=300 \times 300 \mathrm{~mm}^{2}, \mathrm{TBW}=2$ and a simulated heart rate of $60 \mathrm{bpm}$ (For all parameters, please refer to Appendix B, Table 2). T1 was measured in regions of interest (ROI) drawn manually in each tube and mean values in the ROI were compared between SMS and conventional MOLLI.

\subsubsection{AM vs WM}

SMS RF pulses are characterized by highly temporally modulated waveforms. Rapid phase or frequency modulation can be extremely demanding on the performance of RF hardware. Wong [34] showed that the peak of the modulation function can be minimized by adding numerically optimized phase offsets, referred in the following as WM. WM optimisation will usually produce complex-valued RF pulses with rapid modulation of both amplitude and phase components, which is very requiring on MRI RF hardware. Designing equivalent pulses that 
contain only amplitude modulation (real-valued waveforms) [35] can effectively mitigate this issue by limiting modulation on a single dimension. As such, AM pulses rendering has higher fidelity than WM.

For reproducing the unfavourable conditions of SMS in CMR, a tube of mayonnaise was chosen to simulate the epidermal fat and placed on top of the ACR phantom representing the targeted field-of-view (Figure 2.A). AM and WM SMS3-bSSFP images were acquired (For all parameters, please refer to Appendix B, Table 3) with two slices placed inside the phantom and the third slice placed in the signal void corresponding to the plastic casing of the phantom under the mayonnaise tube. The whole first slice was used to quantify the intensity of the side-lobe artefact (Figure 2.A). The intensity of the imaging artefact was normalized by the intensity of one identical slice acquired in the mayonnaise tube. The time-bandwidth product (TBW) being a critical parameter for RF pulse rendering, TBW values of 1, 2 and 3 were tested for their impact on side-lobe artefacts. The RF chain raster time of $1 \mu \mathrm{s}$ which led to 1000 points.

\subsection{In-vivo experiments}

SMS3-bSSFP images were acquired with WM and AM RF design optimizations. TBW was varied from 1 to 3 (For all parameters, please refer to Appendix B, Table 4). The purpose was to compare the two techniques by evaluating the artefact signal obtained. TBW value decrease allowed to soften the pulse profile to reduce the severity of side-lobes artefacts.

Native SMS3-T1 maps were acquired using the 5(3)3 MOLLI scheme. Slice gap was fixed such as to provide simultaneous acquisition of the three levels of basal, mid-ventricular and apical slices (typical gap of $250 \%$ of the slice thickness, i.e. $20 \mathrm{~mm}$ ). 15 minutes after contrast injection, further images were acquired using the same parameters, but with the 4(1)3(1)2 MOLLI scheme [1]. Both modulations $\mathrm{WM}$ and AM were tested pre- and post-contrast. Conventional MOLLI with no SMS acceleration (SMS1-T1) was also performed at the three slice levels (For all parameters, please refer to Appendix B, Table 5). 


\subsection{Image reconstruction}

Image reconstruction including SMS unaliasing was performed using the Gadgetron framework [38]. SMS unaliasing was performed using Split-Slice-GRAPPA [39], in the following named SSG, with leak-block reconstruction. The algorithm was implemented in Matlab (R2016a, Mathworks, Navick, MA) with an empirically optimized kernel size of $5 \times 3$ and a Tikhonov regularization of $10^{-5}$. In-plane acceleration was further reconstructed using L1-eSPIRiT as implemented in BART [40], [41] with Wavelet regularizations (Daubechies, 3 levels) and a 10-

${ }^{3}$ regularization. The custom reconstruction pipeline was embedded in a Matlab "gadget" for direct image reconstruction at the console.

\subsection{In vivo image analysis}

T1 values were obtained by placing ROI on the 16 segments of the left-ventricular (LV) myocardium, over the three slices base, mid and apex, defined by the American Heart Association [42]. In both AM-SMS3-T1 and WM-SMS3-T1, the presence of positive banding artefacts in the myocardium served to identify corrupted segments. The ROI that were placed in the corrupted segments were then removed for a second analysis to isolate the effect of outlier values due to positive banding artefacts.

\subsection{Statistical analysis}

Statistical analysis was performed using GraphPad Prism 7.0a. All values are presented as mean \pm standard deviation (SD). For comparing T1 values between conventional MOLLI and SMS-MOLLI, paired Student's tests were used in addition to a Bland-Altman test to compare the different techniques. Significance was set at P-values $<0.05$. 


\section{Results}

\subsection{Phantom experiments}

\subsubsection{SNR and T1 evaluation}

The highest SNR was obtained with the highest slice thickness (highest SNR for AM $=124 \pm 21$; highest SNR for $\mathrm{WM}=124 \pm 23$ ) (Figure 3.A) and slice gap (highest $\mathrm{SNR}$ for $\mathrm{AM}=92 \pm 5$; highest SNR for $\mathrm{WM}=100 \pm 10$ ) (Figure 3.B), and the lowest $\mathrm{BW}$ tested. There was no difference in SNR between WM-SMS3 and AM-SMS3, but the mean SNR value dropped from $118 \pm 38$ without SMS to $66 \pm 26$ using SMS (AM and WM) when the slice thickness varied. There was also a drop in SNR value when the slice gap varied, from $130 \pm 17$ without SMS to $46 \pm 20$ with SMS (AM and WM). In general, when using SMS, there was $45 \%$ loss of SNR with slice thickness variation, and $64 \%$ loss when the slice gap varied. In the case of acquisitions using standard parameters for cardiac T1 mapping (slice thickness $=8 \mathrm{~mm}$, slice gap $=20 \mathrm{~mm}, \mathrm{BW}=800 \mathrm{~Hz}$ and AM optimization), there is $50 \%$ loss of SNR using SMS.

In terms of $\mathrm{T} 1$ quantification, there was no significant difference when comparing SMS1-T1 and SMS3-T1 values (Fig 4.). SMS3-T1 values were also correlated to the values of the calibrated Agarose tubes $\left(\mathrm{R}^{2}=0.99\right)$ (Fig 4. D). Bland -Altman bias of SMS1-T1 compared to the calibrated $\mathrm{T} 1$ values was $-7.1 \pm 3.5 \%$ with $95 \%$ limits of agreement from $-14.1 \%$ to $-0.15 \%$. When comparing WM-SMS3-T1 values to the vendor provided T1 values, the bias was $1.3 \pm 1.9 \%$ with $95 \%$ limits of agreement from $-4.9 \%$ to $2.4 \%$. In terms of AM-SMS3-T1 values vs. calibrated T1 values, Bland-Altman bias was $-7.2 \pm 3.9 \%$ with $95 \%$ limits of agreement from $-14.9 \%$ to $0.4 \%$. The Bland-Altman analysis between the calibrated T1 values and, SMS1-T1 and both WM-SMS3-T1 and AM-SMS3-T1 values showed that SMS-MOLLI slightly underestimated calibrated T1 values (Fig 4. D) 


\subsubsection{AM vs WM}

As expected with WM, one can clearly see the signal leakage of the tube of fat in the first slice that was positioned outside the phantom and that should not contain signal (Figure 2.B). The positive bandings occurring with the WM can be further amplified by the presence of epidermal fat surrounding the targeted FOV in the apex-base dimension as shown in Appendix A. This artefact can also be seen on the other slices because of the inability of the reconstruction algorithm to separate it from the targeted slices. When using AM, the artefact intensity of the non-targeted slice (fat tube) was significantly reduced on the three slices (Figure 2.B). The two empty slices acquired using AM and WM were compared in terms of artefact signal. There was a significant difference between the two (WM slice: $27.5 \pm 78.8$, AM slice: $17.1 \pm 42.5$, $\mathrm{P}<0.0001)$ showing the efficiency of the $\mathrm{AM}$ technique in artefact reduction $(38 \%$ artefact signal reduction).

\subsection{In-vivo studies}

\subsubsection{AM vs WM}

SMS3-bSSFP images obtained using WM showed slice signal leakage artefacts (Figure 5.A top row) over all slices (basal, median and apical). When using AM optimization, artefacts were either considerably reduced or completely disappeared (Figure 5.A bottom row). With TBW=1, moderately modulated pulse profile led to partial volume effects but resulted in fewer phase variations, and thus facilitate rendering by hardware, such that no signal leakage artefacts can be observed. Increasing TBW to 2 and further to 3 improved slice profile definition as expected, limiting partial volume effect. Higher TBW corresponds to faster RF modulation which implied increased artefacts when using WM (Figure 5.B). Based on these observations, TBW=2 was used for the in-vivo T1 mapping experiments. 


\subsubsection{T1 quantification}

The AM technique was able to decrease the number of corrupted segments in comparison to $\mathrm{WM}$ in the $\mathrm{T} 1$ maps (on average over the 10 volunteers, $5 \pm 3$ and $3 \pm 2$ corrupted segments out of 16 for WM and AM, respectively) (Figure 6). As shown in Figure 7, there was a significant difference between SMS1 and SMS3 native T1 values in the myocardium (SMS1$\mathrm{T} 1=935.5 \pm 36.1 \mathrm{~ms} ; \mathrm{AM}-\mathrm{SMS} 3-\mathrm{T} 1=1050.2 \pm 116.2 \mathrm{~ms} ; \mathrm{P}=0.005)$. However, native $\mathrm{T} 1$ values were equivalent when the corrupted segments were excluded from the analysis (SMS1$\mathrm{T} 1=935.5 \pm 36.1 \mathrm{~ms} ; \mathrm{AM}-\mathrm{SMS} 3-\mathrm{T} 1=933.8 \pm 50.2 \mathrm{~ms} ; \mathrm{P}=0.436) . \quad \mathrm{T} 1$ values in the left-ventricular blood was not significantly different either (SMS1-T1=1475.4 $\pm 35.9 m s ; \quad$ AM-SMS3$\mathrm{T} 1=1452.5 \pm 70.3 \mathrm{~ms} ; \mathrm{P}=0.515)$. Post-gadolinium AM-SMS3-T1 values were not different from the corresponding single-slice values in the myocardium, with and without the corrupted segments (all segments included: SMS1-T1=556.0 $\pm 19.7 \mathrm{~ms} ;$ AM-SMS3-T1=577.9 $\pm 76.3 \mathrm{~ms}$; $\mathrm{P}=0.511 ; \quad$ corrupted segments excluded: $\quad \mathrm{SMS1-T1}=556.0 \pm 19.7 \mathrm{~ms} ; \quad$ AM-SMS3$\mathrm{T} 1=521.3 \pm 28.1 \mathrm{~ms} ; \mathrm{P}=0.626)$, as well as in the LV blood pool $(\mathrm{SMS} 1-\mathrm{T} 1=478 \pm 65.1 \mathrm{~ms}$; AMSMS3-T1 $=447.8 \pm 81.5 \mathrm{~ms} ; \mathrm{P}=0.085)$. For more in depth data analysis, Appendix $\mathrm{C}$ was added to provide mean $\pm \mathrm{SD} \mathrm{T} 1$ values for each one of the $16 \mathrm{LV}$ myocardium segments over the 10 healthy volunteers. Native SMS1-T1, post injection SMS1-T1, native AM-SMS3-T1 with and without corrupted segments, and post injection AM-SMS3-T1 also with and without corrupted segments was included.

Bland-Altman analysis over the 10 volunteers for the comparison between the SMS1-T1 and AM-SMS3-T1 techniques is reported in Table 1. Excluding the corrupted segments from the analysis of the myocardium T1 values, highly reduced the Bland-Altman bias when comparing native SMS1-T1 and AM-SMS3-T1 values (bias with all segments $=-11.3 \pm 9.8 \%$; bias without corrupted segments $=1.5 \pm 5.4 \%$, and postcontrast SMS1-T1 and AM-SMS3-T1 values (bias with all segments $-3.0 \pm 12.5 \%$; bias without corrupted segments $=0.7 \pm 3.1 \%$ ). 


\section{Discussion}

In this study, we showed that proposed Blipped-CAIPIRINHA-SMS-MOLLI allowed to extend spatial coverage from single-slice MOLLI to 3 slices, covering the three levels of the left ventricle. Resulting T1 values showed excellent correlation to reference T1 values and singleslice MOLLI values when tested on a calibrated phantom. However, side-lobes artefacts from Wong-modulated RF pulses were identified. Alternative RF modulation using amplitude-only optimization reduced these artefacts and improved T1 maps quality. Results from 10 healthy volunteers also showed in vivo artefacts reduction and artefacts-free T1 values obtained were comparable to single-slice MOLLI values.

Due to the nature of bSSFP sequences, where both longitudinal and transverse magnetization need to be maintained in a steady state, the use of phase cycling [43] for SMS implementations is very challenging, hence the interest of using gradient-blipped CAIPIRINHA to facilitate the separation of simultaneously excited slices. In this work, we present a SSFP implementation of the blipped-CAIPIRINHA SMS technique initially proposed by Setsompop et al. [32] for EPI, in the context of the MOLLI sequence for CMR T1 mapping. A unique challenge in SMSbSSFP imaging is the dramatic enhancement of low flip-angle excitations corresponding to undesired side-lobe excitation [36]. Indeed, the difficulty for MRI hardware to produce short $(<1 \mathrm{~ms})$ rapidly modulated multiband-RF pulses translates into excitation imperfections. These imperfections can be negligible in spoiled gradient-echo sequences, but they have a severe impact when occurring in bSSFP where they cause spatially localized positive banding artefacts that can leak across the simultaneously excited slices. These positive banding signals are not resolved by the SMS reconstruction and eventually pollute one or several of the reconstructed slices.

In the context of longer RF pulses with numerous bands $(\mathrm{N}=6)$, Abo-Seada et al. [35] proposed a solution to mitigate SMS excitation imperfections based on amplitude-only modulated RF 
design. Here, this method was adapted to bSSFP with shorter pulses and fewer bands $(\mathrm{N}=3)$ and compared to standard phase optimization techniques [34] intended for peak RF power reduction. Given the reduction of artefacts provided by AM-SMS-bSSFP, we also evaluated reproducibility and reliability of SMS-accelerated MOLLI T1 mapping and compared them to a conventional MOLLI sequence.

The obtained results indicate that positive banding artefacts in the context of blippedCAIPIRINHA SMS-bSSFP can be reduced using the AM technique. Reduction of similar artefacts using AM compared to WM has also been demonstrated with different RF duration and resolution [35]. Applied to the MOLLI sequence, blipped-CAIPIRINHA SMS acceleration retains T1 accuracy compared to conventional MOLLI or literature values [13], for both myocardium and blood. By extension, CAIPIRINHA SMS-bSSFP has the potential to accelerate other T1 mapping techniques [15], [19], [28] as well as multiple CMR applications, including T2 mapping, cine as well as dynamic real-time imaging in the body. In this context, the use of AM multi-band RF modulation will be key to avoid artefacts as observed presently.

\section{$\underline{\text { Limitations }}$}

Several potential alternative solutions for handling positive banding artefacts were not tested here. First, conventional CAIPIRINHA slice-undersampling (shifts of $2 \pi /$ Nslices) could be substituted to displace the artefacts as the slice shifts are adjusted. This solution remains to be adjusted per acquisition, and the intensity and size of the artefacts would penalize the clinical usefulness of the acceleration. Second, the Gradient-controlled local Larmor adjustment (GCLOLA) approach proposed by Stäb and Speier [30] could be a candidate to obtain SMS acceleration instead of blipped-CAIPIRINHA. GC-LOLA is known to perturbate the frequency response from the bSSFP readout. This perturbation could potentially mitigate the enhancement of positive banding in side-lobe excitation slices. Exploring the occurrence of positive banding in GC-LOLA would need to consider the particular conditions of our study: the number of 
bands $(\mathrm{N}=3)$ was higher than that reported with GC-LOLA $(\mathrm{N}=2)$, and we focussed on singleshot bSSFP for MOLLI, which might be more sensitive to side-lobes excitations than steadystate bSSFP as employed in GC-LOLA cine. A third solution, as proposed by Landes et al.[36], is to calibrate for these extra excited slices. By acquiring calibration data from these extra slices, the positive banding signals can be resolved at the SMS reconstruction stage and completely removed from the targeted slices. This solution assumes that the side-lobe positions can be identified, and that additional data are acquired prior to imaging. The first condition was satisfied by Landes et al. using a thorough measurement of the exact RF excitation envelope using gradient reversal acquisition [44]. The second condition would require extra calibration data corresponding to side-lobes positions at the beginning of the SMS acquisition, increasing the potential for misalignment between the extended calibration acquisition and the accelerated imaging acquisition [27], as well as lengthening the total acquisition time, thus reducing the benefits from the SMS acceleration. Fourth, Landes et al. proposed more recently [45] to tune the RF design to purposely reduce side-lobes excitation by an iterative process between RF exact shape measurement using GRATER [44] and RF design. This solution is tedious and can only fix the design for one RF pulse at a time, but it has the potential to reduce even further the side-lobe excitation than the proposed AM-only RF design.

\section{Conclusions}

Consensus-driven 3-slices MOLLI T1 mapping can be shortened to a single breath-hold using SMS-bSSFP. To reduce artefacts, amplitude-modulated RF excitations should be preferred albeit residual artefacts remain. Indeed, from the result that side-lobes excitations artefacts were linked to Wong RF phase-modulation, AM-SMS-bSSFP was able to reduce side-lobes artefacts considerably. Additionally, the proposed solution to exploit amplitude-only modulated multiband RF design is highly versatile, easy to reproduce and offers solid foundations to pursue SMS-bSSFP evaluation for accelerated cardiac MRI. Complementary technical 
improvements, such as an improved SMS reconstruction algorithm to isolate targeted signals [46], may further benefit SMS-accelerated MOLLI and contribute to reaching clinical reliability in the future. 


\section{Declarations}

\section{Ethics approval and consent to participate}

The COFLORES clinical trial was registered under the number NCT02848703

(www.clinicaltrials.gov). The Committee for the Protection of Persons (CPP) gave consent to the study on April 2016. All participants enrolled a CMR examination after providing an informed consent.

Declarations of interest: none

\section{Funding}

Fondation pour la Recherche Médicale, Grant/Award Number: DBS20140930772; Agence Nationale de la Recherche, Grant/ Award Number: ANR-14-CE17-0016.

This work was performed by a laboratory member of France Life Imaging network (grant ANR-11-INBS-0006), on the platform 7T-AMI, a French "Investissements d'Avenir" programme" (grant ANR-11-EQPX-0001).

\section{Authors contribution}

ZB and SR designed and implemented the sequence and the reconstruction.

ZB, SR, FK, TT, LP participated in the experiments.

ZB and SR analyzed and interpreted the phantom and volunteers' data.

All authors read and approved the final manuscript.

\section{Acknowledgements}

The authors express their gratitude towards the CRMBM support team, notably V. Gimenez, C. Coste and P. Viout.

\section{Availability of data and materials}

Data and materials, including the sequence, reconstruction and the datasets generated during and/or analysed during the current study are available from the corresponding author on reasonable request. 


\section{Figure legends}

Fig. 1. Blipped CAIPIRINHA SMS-bSSFP. GZ Rewind gradients of the bSSFP are transformed into blips by adding and subtracting phase offsets at each TR. RF modulation is preserved, and bSSFP frequency response as well.

Fig. 2. Comparison between Wong modulated, and amplitude modulated SMS3-bSSFP images. (A) SMS3-bSSFP images were acquired in a water phantom with the proximity of fat (mayonnaise) to mimic in vivo CMR context. The first slice was placed in the void between the phantom and the fat to enhance artefacts. (B) WM-SMS3-bSSFP leads to spurious side-lobes excitations that are not calibrated for and lead to positive artefacts in reconstructed slices. Change of RF modulation using AM reduces side-lobes artefacts (WM slice: 27.5 578.8 , AM slice: $17.1 \pm 42.5, \mathrm{P}<0.0001$ corresponding to $38 \%$ artefact reduction). WM, Wong modulation; AM, amplitude modulation.

Fig. 3. SNR variation maps. (A): slice thickness and bandwidth variation (slice gap $=48 \mathrm{~mm}$ ). (B): slice gap and bandwidth variation (slice thickness $=6 \mathrm{~mm}$ ). Highest SNR values were obtained with higher slice thickness and slice gap, and lower bandwidth.

WM, Wong modulation; AM, amplitude modulation.

Fig. 4. T1 maps of calibrated Agarose gel tubes using SMS3 and SMS1 MOLLI. (A) Regions of interest, with the corresponding tube number, used to get T1 values. (B) Bar plot of the calibrated values of each tube and those obtained using SMS1-T1, WM-SMS3-T1 and AMSMS3-T1. (C) SMS1-T1(left) and AM-SMS3-T1(right) maps of the middle slice. (D) BlandAltman analysis comparing the different MOLLI techniques (SMS1-T1, WM-SMS3-T1 and AM-SMS3-T1) to the vendor provided T1 values of the Agarose tubes. The three techniques underestimate the theoretical T1 values, most probably due to the MOLLI estimation model, with no significant difference and small bias between SMS1-T1 values of the conventional 
MOLLI sequence, and both WM-SMS3-T1 and AM-SMS3-T1 values of the BlippedCAIPIRINHA-SMS-MOLLI sequence. WM, Wong modulation; AM, amplitude modulation. Fig. 5. Comparison between WM and AM for SMS3 Blipped-CAIPIRINHA bSSFP. (A) comparison over the three slices base, mid and apex. (B) TBW value effect on images with WM and AM optimization over the basal slice. The white arrows show the signal leakage artefacts when using WM that completely disappear with AM. WM, Wong modulation; AM, amplitude modulation; TBW; product Time-Band Width.

Fig. 6. T1 maps over base, mid and apex using blipped-CAIPIRINHA SMS-MOLLI. From top to bottom: native SMS1-T1 maps using conventional MOLLI, native AM-SMS3-T1 maps using SMS-MOLLI, postcontrast (15 $\mathrm{min})$ SMS1-T1 maps and postcontrast (15 $\mathrm{min})$ AMSMS3-T1. AM, amplitude modulation.

Fig. 7. Box-and-whisker plots of the mean native $\mathrm{T} 1$ and postcontrast $\mathrm{T} 1$ values in the myocardium and the blood using conventional MOLLI and AM-SMS3-MOLLI (with and without the artefact-corrupted LV segments).

\section{Tables legends}

Table 1. Bland-Altman analysis for comparison between SMS1-T1 and AM-SMS3-T1 techniques over the 10 volunteers. 


\section{References}

[1] P. Kellman and M. S. Hansen, "T1-mapping in the heart: accuracy and precision," Journal of Cardiovascular Magnetic Resonance, vol. 16, no. 1, p. 2, 2014, doi: 10.1186/1532-429X-16-2.

[2] V. O. Puntmann, E. Peker, Y. Chandrashekhar, and E. Nagel, "T1 Mapping in Characterizing Myocardial Disease: A Comprehensive Review," Circulation Research, vol. 119, no. 2, pp. 277-299, Jul. 2016, doi: 10.1161/CIRCRESAHA.116.307974.

[3] J. R. Burt, S. L. Zimmerman, I. R. Kamel, M. Halushka, and D. A. Bluemke, "Myocardial T1 mapping: techniques and potential applications," Radiographics, vol. 34, no. 2, pp. 377-395, Apr. 2014, doi: 10.1148/rg.342125121.

[4] N. Mewton, C. Y. Liu, P. Croisille, D. Bluemke, and J. A. C. Lima, "Assessment of myocardial fibrosis with cardiovascular magnetic resonance," J. Am. Coll. Cardiol., vol. 57, no. 8, pp. 891-903, Feb. 2011.

[5] A. S. Flett et al., "Equilibrium contrast cardiovascular magnetic resonance for the measurement of diffuse myocardial fibrosis: preliminary validation in humans," Circulation, vol. 122, no. 2, pp. 138-144, Jul. 2010, doi: 10.1161/CIRCULATIONAHA.109.930636.

[6] P. Kellman, J. R. Wilson, H. Xue, M. Ugander, and A. E. Arai, "Extracellular volume fraction mapping in the myocardium, part 1: evaluation of an automated method," Journal of Cardiovascular Magnetic Resonance, vol. 14, no. 1, Dec. 2012, doi: 10.1186/1532-429X-14-63.

[7] P. Kellman et al., "Extracellular volume fraction mapping in the myocardium, part 2: initial clinical experience," Journal of Cardiovascular Magnetic Resonance, vol. 14, no. 1, Dec. 2012, doi: 10.1186/1532-429X-14-64.

[8] D. M. Sado et al., "Cardiovascular magnetic resonance measurement of myocardial extracellular volume in health and disease," Heart, vol. 98, no. 19, pp. 1436-1441, Oct. 2012, doi: 10.1136/heartjnl-2012-302346.

[9] M. Ugander et al., "Extracellular volume imaging by magnetic resonance imaging provides insights into overt and sub-clinical myocardial pathology," European Heart Journal, vol. 33, no. 10, pp. 1268-1278, May 2012, doi: 10.1093/eurheartj/ehr481.

[10] T. D. Karamitsos et al., "Noncontrast T1 Mapping for the Diagnosis of Cardiac Amyloidosis," JACC: Cardiovascular Imaging, vol. 6, no. 4, pp. 488-497, Apr. 2013, doi: 10.1016/j.jcmg.2012.11.013.

[11] J. C. Wood et al., "Cardiac Iron Determines Cardiac T2*, T2, and T1 in the Gerbil Model of Iron Cardiomyopathy," Circulation, vol. 112, no. 4, pp. 535-543, Jul. 2005, doi: 10.1161/CIRCULATIONAHA.104.504415.

[12] A. Liu et al., "Gadolinium-Free Cardiac MR Stress T1-Mapping to Distinguish Epicardial From Microvascular Coronary Disease," Journal of the American College of Cardiology, vol. 71, no. 9, pp. 957-968, Mar. 2018, doi: 10.1016/j.jacc.2017.11.071.

[13] D. R. Messroghli, A. Radjenovic, S. Kozerke, D. M. Higgins, M. U. Sivananthan, and J. P. Ridgway, "Modified Look-Locker inversion recovery (MOLLI) for high-resolutionT1 mapping of the heart," Magnetic Resonance in Medicine, vol. 52, no. 1, pp. 141-146, Jul. 2004, doi: 10.1002/mrm.20110.

[14] S. Roujol et al., "Accuracy, Precision, and Reproducibility of Four T1 Mapping Sequences: A Head-to-Head Comparison of MOLLI, ShMOLLI, SASHA, and SAPPHIRE," Radiology, vol. 272, no. 3, pp. 683-689, Sep. 2014, doi: 10.1148/radiol.14140296. 
[15] L. Huang et al., "FASt single-breathhold 2D multislice myocardial T 1 mapping (FAST1) at $1.5 \mathrm{~T}$ for full left ventricular coverage in three breathholds," Journal of Magnetic Resonance Imaging, vol. 51, no. 2, pp. 492-504, Feb. 2020, doi: 10.1002/jmri.26869.

[16] S. K. Piechnik et al., "Shortened Modified Look-Locker Inversion recovery (ShMOLLI) for clinical myocardial T1-mapping at 1.5 and 3 T within a 9 heartbeat breathhold," Journal of Cardiovascular Magnetic Resonance, vol. 12, no. 1, Dec. 2010, doi: 10.1186/1532-429X-12-69.

[17] L. Huang et al., "Fast myocardial $\mathrm{T}_{1}$ mapping using shortened inversion recovery based schemes: Fast Myocardial T 1 Mapping," Journal of Magnetic Resonance Imaging, vol. 50, no. 2, pp. 641-654, Aug. 2019, doi: 10.1002/jmri.26649.

[18] X. Wang, F. Kohler, C. Unterberg-Buchwald, J. Lotz, J. Frahm, and M. Uecker, "Modelbased myocardial T1 mapping with sparsity constraints using single-shot inversionrecovery radial FLASH cardiovascular magnetic resonance," Journal of Cardiovascular Magnetic Resonance, vol. 21, no. 1, Dec. 2019, doi: 10.1186/s12968-019-0570-3.

[19] S. Weingärtner, S. Roujol, M. Akçakaya, T. A. Basha, and R. Nezafat, "Free-breathing multislice native myocardial $\mathrm{T}_{1}$ mapping using the slice-interleaved $\mathrm{T}_{1}$ (STONE) sequence: Multislice IR Myocardial T 1 Mapping," Magnetic Resonance in Medicine, vol. 74, no. 1, pp. 115-124, Jul. 2015, doi: 10.1002/mrm. 25387.

[20] M. S. Nazir et al., "Simultaneous multi slice (SMS) balanced steady state free precession first-pass myocardial perfusion cardiovascular magnetic resonance with iterative reconstruction at 1.5 T," Journal of Cardiovascular Magnetic Resonance, vol. 20, no. 1, Dec. 2018, doi: 10.1186/s12968-018-0502-7.

[21] D. Stäb et al., "High resolution myocardial first-pass perfusion imaging with extended anatomic coverage: High Coverage Myocardial Perfusion MRI," Journal of Magnetic Resonance Imaging, vol. 39, no. 6, pp. 1575-1587, Jun. 2014, doi: 10.1002/jmri.24303.

[22] H. Wang, G. Adluru, L. Chen, E. G. Kholmovski, N. K. Bangerter, and E. V. R. DiBella, "Radial simultaneous multi-slice CAIPI for ungated myocardial perfusion," Magnetic Resonance Imaging, vol. 34, no. 9, pp. 1329-1336, Nov. 2016, doi: 10.1016/j.mri.2016.07.015.

[23] Y. Tian et al., "Feasibility of multiple-view myocardial perfusion MRI using radial simultaneous multi-slice acquisitions," PLOS ONE, vol. 14, no. 2, p. e0211738, Feb. 2019, doi: 10.1371/journal.pone.0211738.

[24] Y. Yang, C. H. Meyer, F. H. Epstein, C. M. Kramer, and M. Salerno, "Whole-heart spiral simultaneous multi-slice first-pass myocardial perfusion imaging," Magnetic Resonance in Medicine, vol. 81, no. 2, pp. 852-862, Feb. 2019, doi: $10.1002 / \mathrm{mrm} .27412$.

[25] M. Barth, F. Breuer, P. J. Koopmans, D. G. Norris, and B. A. Poser, "Simultaneous multislice (SMS) imaging techniques: SMS Imaging," Magnetic Resonance in Medicine, vol. 75, no. 1, pp. 63-81, Jan. 2016, doi: 10.1002/mrm.25897.

[26] F. A. Breuer, M. Blaimer, R. M. Heidemann, M. F. Mueller, M. A. Griswold, and P. M. Jakob, "Controlled aliasing in parallel imaging results in higher acceleration (CAIPIRINHA) for multi-slice imaging," Magnetic Resonance in Medicine, vol. 53, no. 3, pp. 684-691, Mar. 2005, doi: 10.1002/mrm.20401.

[27] S. Rapacchi et al., "Simultaneous multi-slice cardiac cine with Fourier-encoded selfcalibration at 7 Tesla," Magnetic Resonance in Medicine, vol. 81, no. 4, pp. 2576-2587, Apr. 2019, doi: 10.1002/mrm.27593. 
[28] S. Weingärtner et al., "Simultaneous multislice imaging for native myocardial $\mathrm{T}_{1}$ mapping: Improved spatial coverage in a single breath-hold: Multislice Imaging for Myocardial T 1 Mapping," Magnetic Resonance in Medicine, vol. 78, no. 2, pp. 462471, Aug. 2017, doi: 10.1002/mrm.26770.

[29] D. Stäb, C. O. Ritter, F. A. Breuer, A. M. Weng, D. Hahn, and H. Köstler, "CAIPIRINHA accelerated SSFP imaging: CAIPIRINHA Accelerated SSFP Imaging," Magnetic Resonance in Medicine, vol. 65, no. 1, pp. 157-164, Jan. 2011, doi: 10.1002/mrm.22600.

[30] D. Stäb and P. Speier, "Gradient-controlled local Larmor adjustment (GC-LOLA) for simultaneous multislice bSSFP imaging with improved banding behavior," Magnetic Resonance in Medicine, vol. 81, no. 1, pp. 129-139, Jan. 2019, doi: 10.1002/mrm.27356.

[31] J. Duerk, M. Griswold, and K. Dara, "Multi-slice blipped TRUEFISP-CAIPIRINHA," US 20130271128A1, Apr. 12, 2012.

[32] K. Setsompop, B. A. Gagoski, J. R. Polimeni, T. Witzel, V. J. Wedeen, and L. L. Wald, "Blipped-controlled aliasing in parallel imaging for simultaneous multislice echo planar imaging with reduced g-factor penalty," Magnetic Resonance in Medicine, vol. 67, no. 5, pp. 1210-1224, May 2012, doi: 10.1002/mrm.23097.

[33] A. N. Price, L. Cordero-Grande, S. J. Malik, and J. V. Hajnal, "Simultaneous multislice imaging of the heart using multiband balanced SSFP with blipped-CAIPI," Magnetic Resonance in Medicine, vol. 83, no. 6, pp. 2185-2196, 2020, doi: 10.1002/mrm.28086.

[34] E. Wong, "Optimized Phase Schedules for Minimizing Peak RF Power in Simultaneous Multi-Slice RF Excitation Pulses," Proceedings of the 20th Annual Meeting of ISMRM, vol. 20, 2012.

[35] S. Abo Seada, A. N. Price, J. V. Hajnal, and S. J. Malik, "Optimized amplitude modulated multiband RF pulse design: Optimized Amplitude Modulated Multiband RF Pulse Design," Magnetic Resonance in Medicine, vol. 78, no. 6, pp. 2185-2193, Dec. 2017, doi: 10.1002/mrm.26610.

[36] V. Landes, "Practical implementation of SMS bSSFP in the Heart," Proceedings of the 26th Annual Meeting of ISMRM, vol. 26, Jun. 2018.

[37] "Phantom Test Guidance for Use of the Large MRI Phantom of the ACR." American College of Radiology, Apr. 17, 2018, [Online]. Available:

https://www.acraccreditation.org//media/ACRAccreditation/Documents/MRI/LargePhantomGuidance.pdf.

[38] M. S. Hansen and T. S. Sørensen, "Gadgetron: An open source framework for medical image reconstruction: Gadgetron," Magnetic Resonance in Medicine, vol. 69, no. 6, pp. 1768-1776, Jun. 2013, doi: 10.1002/mrm.24389.

[39] S. F. Cauley, J. R. Polimeni, H. Bhat, L. L. Wald, and K. Setsompop, "Interslice leakage artifact reduction technique for simultaneous multislice acquisitions: Interslice Leakage Artifact Reduction Technique," Magnetic Resonance in Medicine, vol. 72, no. 1, pp. 93102, Jul. 2014, doi: 10.1002/mrm.24898.

[40] M. Uecker et al., "Bart: Version 0.2.09." Zenodo, Oct. 07, 2015, doi: 10.5281/zenodo.31907.

[41] M. Uecker et al., "ESPIRiT-an eigenvalue approach to autocalibrating parallel MRI: Where SENSE meets GRAPPA," Magnetic Resonance in Medicine, vol. 71, no. 3, pp. 990-1001, Mar. 2014, doi: 10.1002/mrm.24751.

[42] M. D. Cerqueira et al., "Standardized myocardial segmentation and nomenclature for tomographic imaging of the heart. A statement for healthcare professionals from the 
Cardiac Imaging Committee of the Council on Clinical Cardiology of the American Heart Association," J Nucl Cardiol, vol. 9, no. 2, pp. 240-245, Apr. 2002.

[43] F. A. Breuer et al., "Controlled aliasing in volumetric parallel imaging (2D CAIPIRINHA)," Magnetic Resonance in Medicine, vol. 55, no. 3, pp. 549-556, Mar. 2006, doi: 10.1002/mrm.20787.

[44] V. L. Landes and K. S. Nayak, "Simple method for RF pulse measurement using gradient reversal: RF Pulse Measurement Using Gradient Reversal," Magnetic Resonance in Medicine, vol. 79, no. 5, pp. 2642-2651, May 2018, doi: $10.1002 / \mathrm{mrm} .26920$.

[45] V. L. Landes and K. S. Nayak, "Iterative correction of RF envelope distortion with GRATER-measured waveforms," Magnetic Resonance in Medicine, vol. 83, no. 1, pp. 188-194, 2020, doi: 10.1002/mrm.27930.

[46] S. Park, L. Chen, A. Beckett, and D. A. Feinberg, "Virtual slice concept for improved simultaneous multi-slice MRI employing an extended leakage constraint," Magnetic Resonance in Medicine, vol. 82, no. 1, pp. 377-386, Jul. 2019, doi: 10.1002/mrm.27741.

[47] N. Kawel-Boehm et al., "Normal values for cardiovascular magnetic resonance in adults and children," Journal of Cardiovascular Magnetic Resonance, vol. 17, no. 1, p. 29, Apr. 2015, doi: 10.1186/s12968-015-0111-7.

[48] P. A. Bottomley, T. H. Foster, R. E. Argersinger, and L. M. Pfeifer, "A review of normal tissue hydrogen NMR relaxation times and relaxation mechanisms from 1-100 MHz: Dependence on tissue type, NMR frequency, temperature, species, excision, and age: Review Article: NMR relaxation in tissue," Medical Physics, vol. 11, no. 4, pp. 425448, Jul. 1984, doi: 10.1118/1.595535. 


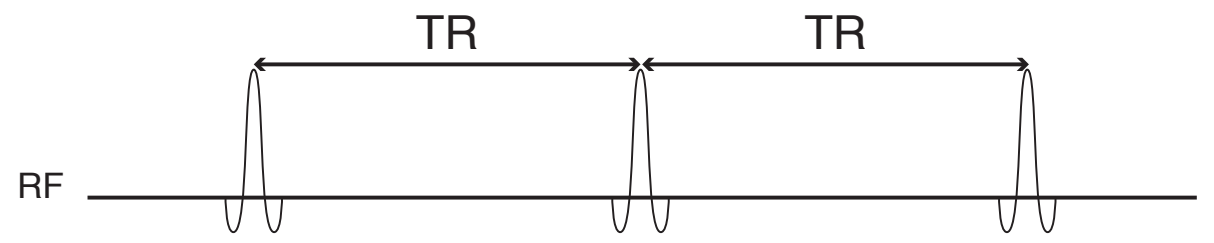

$\mathrm{GZ}$
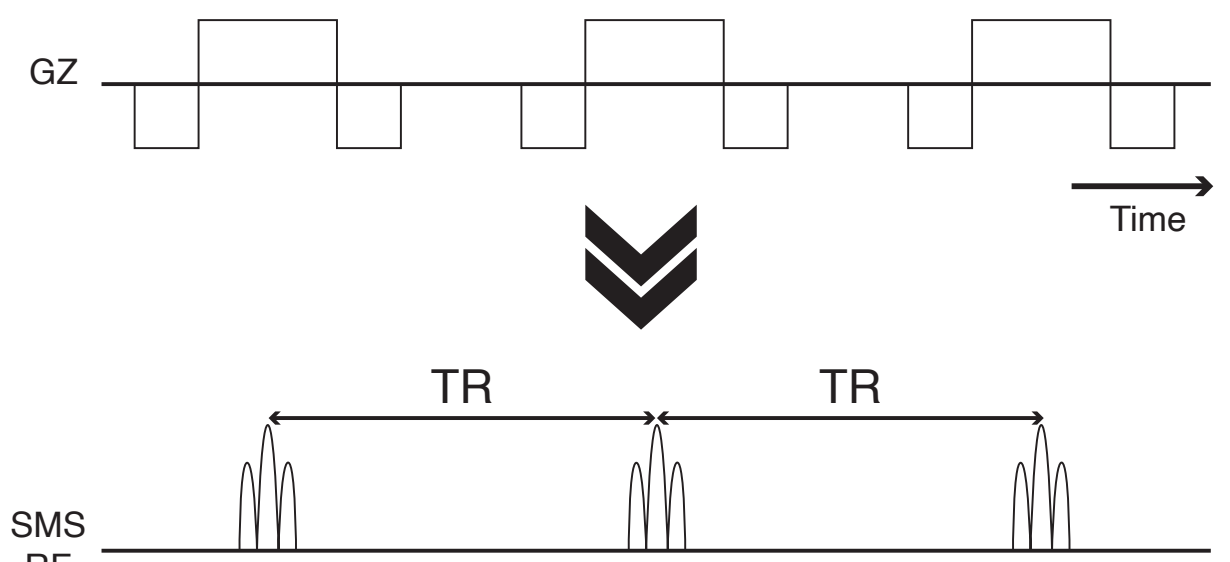

$\mathrm{RF}$

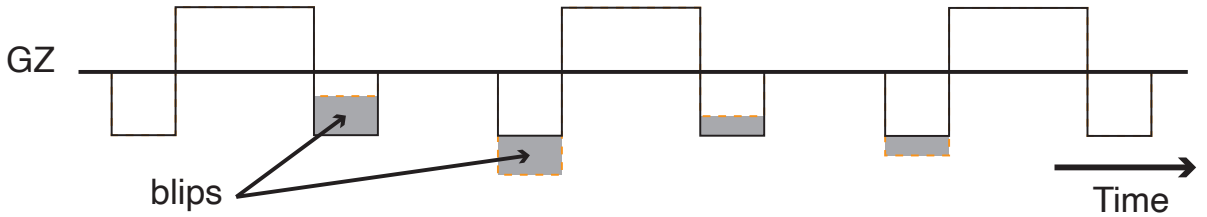

Fig. 1. Blipped CAIPIRINHA SMS-bSSFP. GZ Rewind gradients of the bSSFP are transformed into blips by adding and subtracting phase offsets at each TR. RF modulation is preserved, and bSSFP frequency response as well.
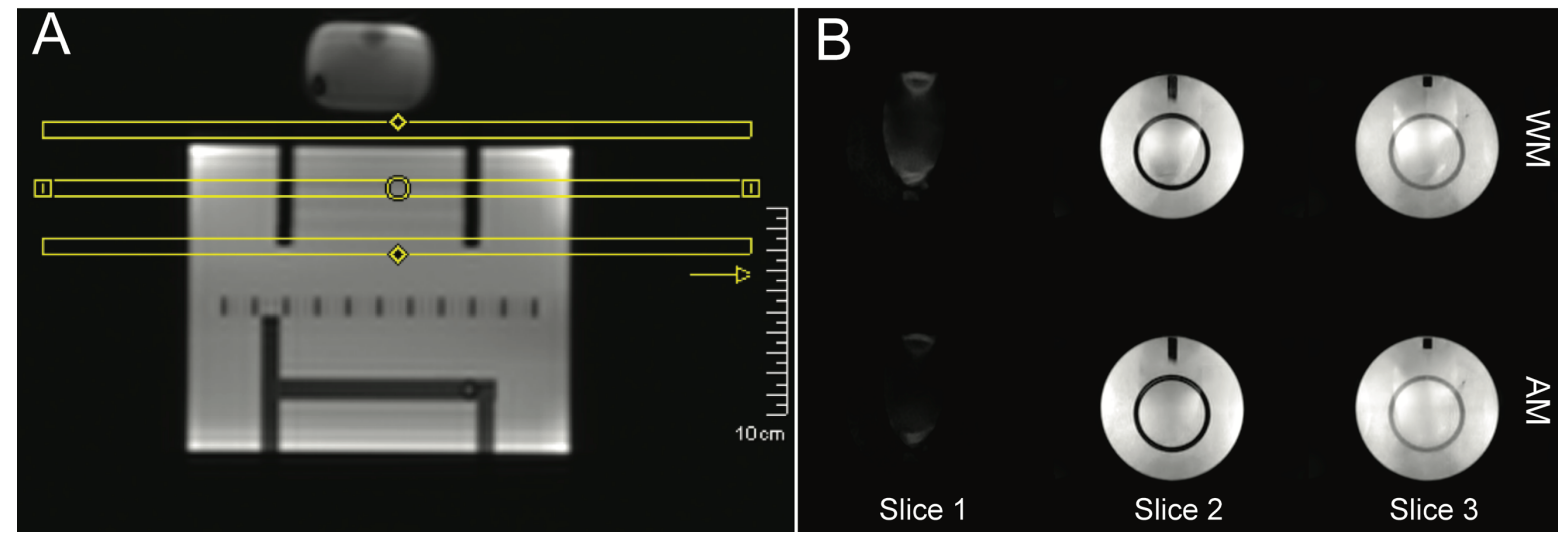

Fig. 2. Comparison between Wong modulated, and amplitude modulated SMS3-bSSFP images. (A) SMS3-bSSFP images were acquired in a water phantom with the proximity of fat (mayonnaise) to mimic in vivo CMR context. The first slice was placed in the void between the phantom and the fat to enhance artefacts. (B) WM-SMS3-bSSFP leads to spurious side-lobes excitations that are not calibrated for and lead to positive artefacts in reconstructed slices. Change of RF modulation using AM reduces side-lobes artefacts (WM slice: $27.5 \pm 78.8$, AM slice: $17.1 \pm 42.5, \mathrm{P}<0.0001$ corresponding to $38 \%$ artefact reduction). WM, Wong modulation; AM, amplitude modulation. 


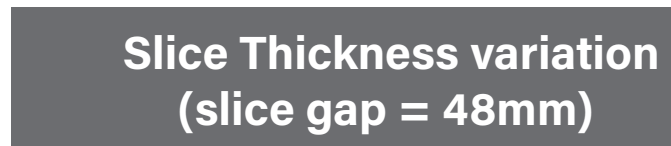

A
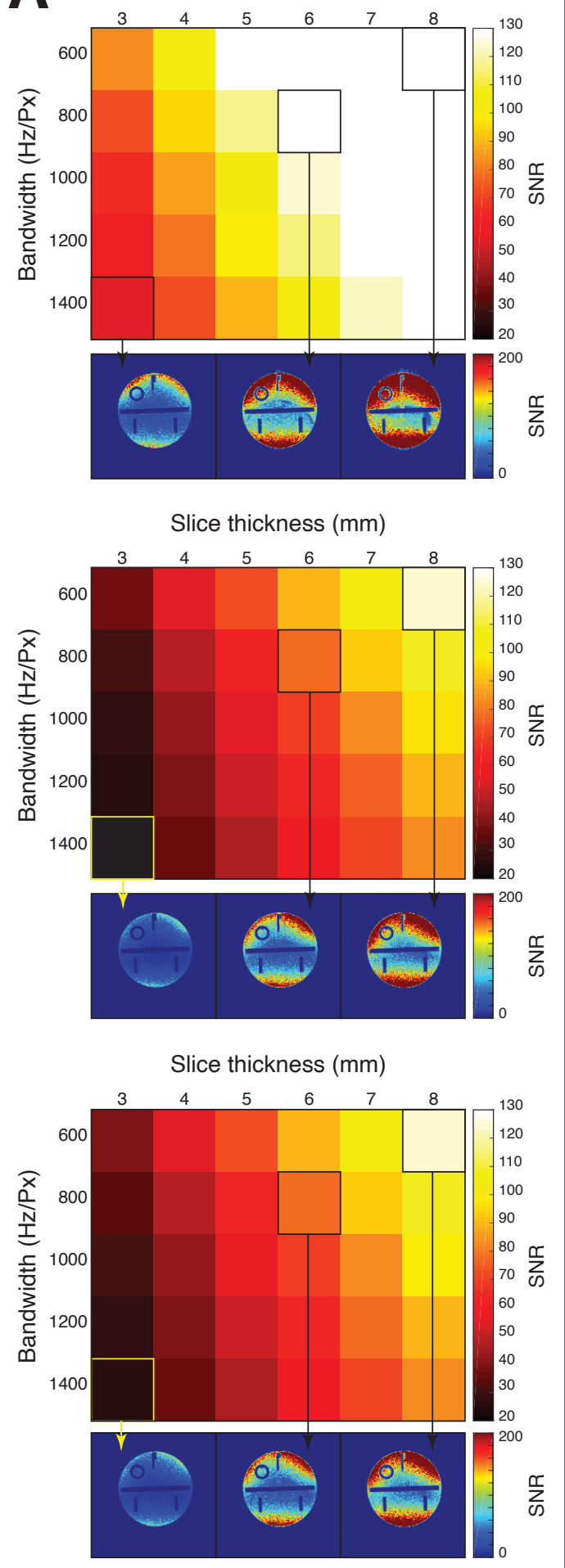

\section{Slice gap variation (slice thickness $=6 \mathrm{~mm}$ )}

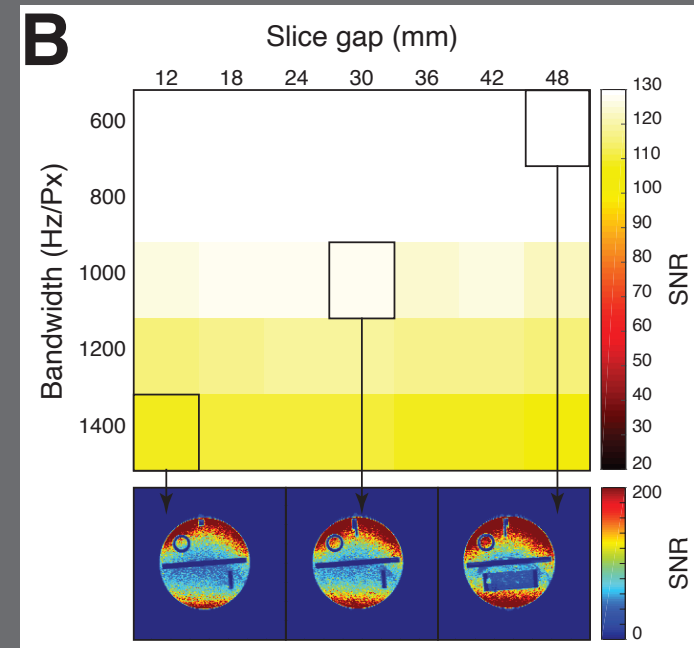

Slice gap $(\mathrm{mm})$

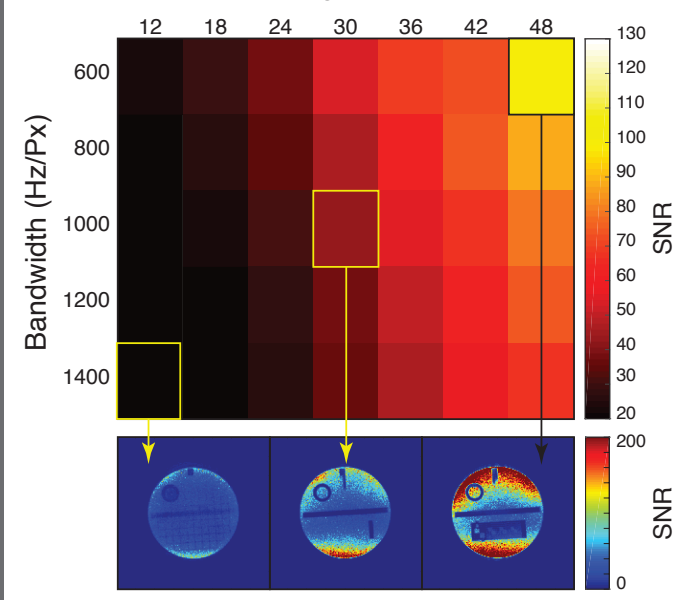

Slice gap $(\mathrm{mm})$

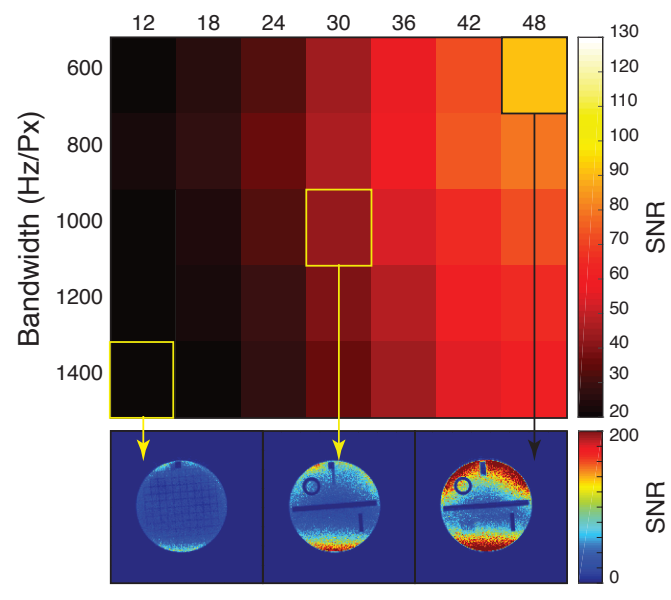

Fig. 3. SNR variation maps. (A): slice thickness and bandwidth variation (slice gap $=48 \mathrm{~mm}$ ). (B): slice gap and bandwidth variation (slice thickness $=6 \mathrm{~mm}$ ). Highest SNR values were obtained with higher slice thickness and slice gap, and lower bandwidth. WM, Wong modulation; AM, amplitude modulation. 

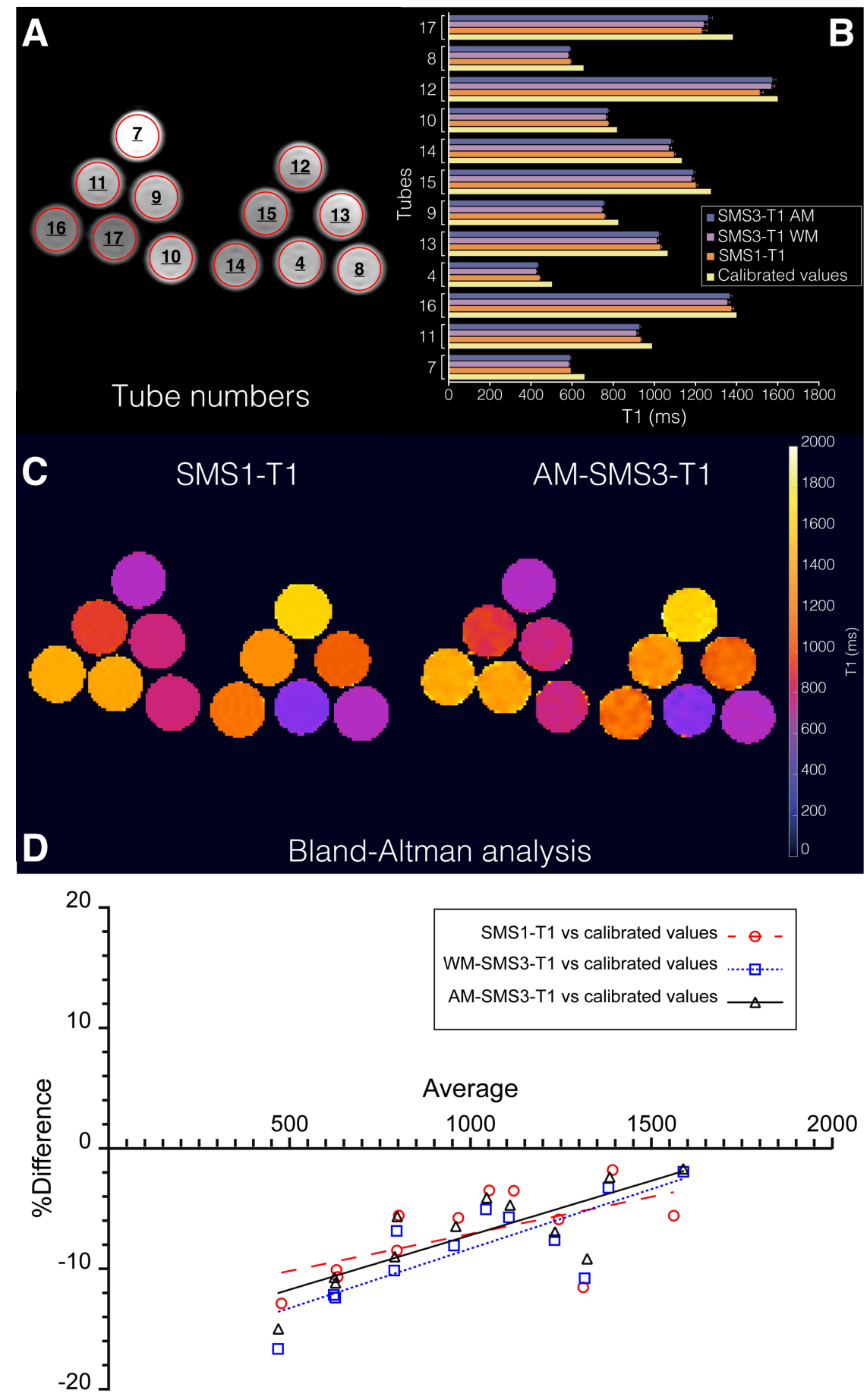

Fig. 4. T1 maps of calibrated Agarose gel tubes using SMS3 and SMS1 MOLLI. (A) Regions of interest, with the corresponding tube number, used to get T1 values. (B) Bar plot of the calibrated values of each tube and those obtained using SMS1-T1, WM-SMS3-T1 and AM-SMS3-T1. (C) SMS1-T1(left) and AM-SMS3-T1(right) maps of the middle slice. (D) Bland-Altman analysis comparing the different MOLLI techniques (SMS1-T1, WM-SMS3-T1 and AM-SMS3-T1) to the vendor provided T1 values of the Agarose tubes. The three techniques underestimate the theoretical $\mathrm{T} 1$ values, most probably due to the MOLLI estimation model, with no significant difference and small bias between SMS1-T1 values of the conventional MOLLI sequence, and both WM-SMS3-T1 and AM-SMS3-T1 values of the BlippedCAIPIRINHA-SMS-MOLLI sequence. WM, Wong modulation; AM, amplitude modulation. 


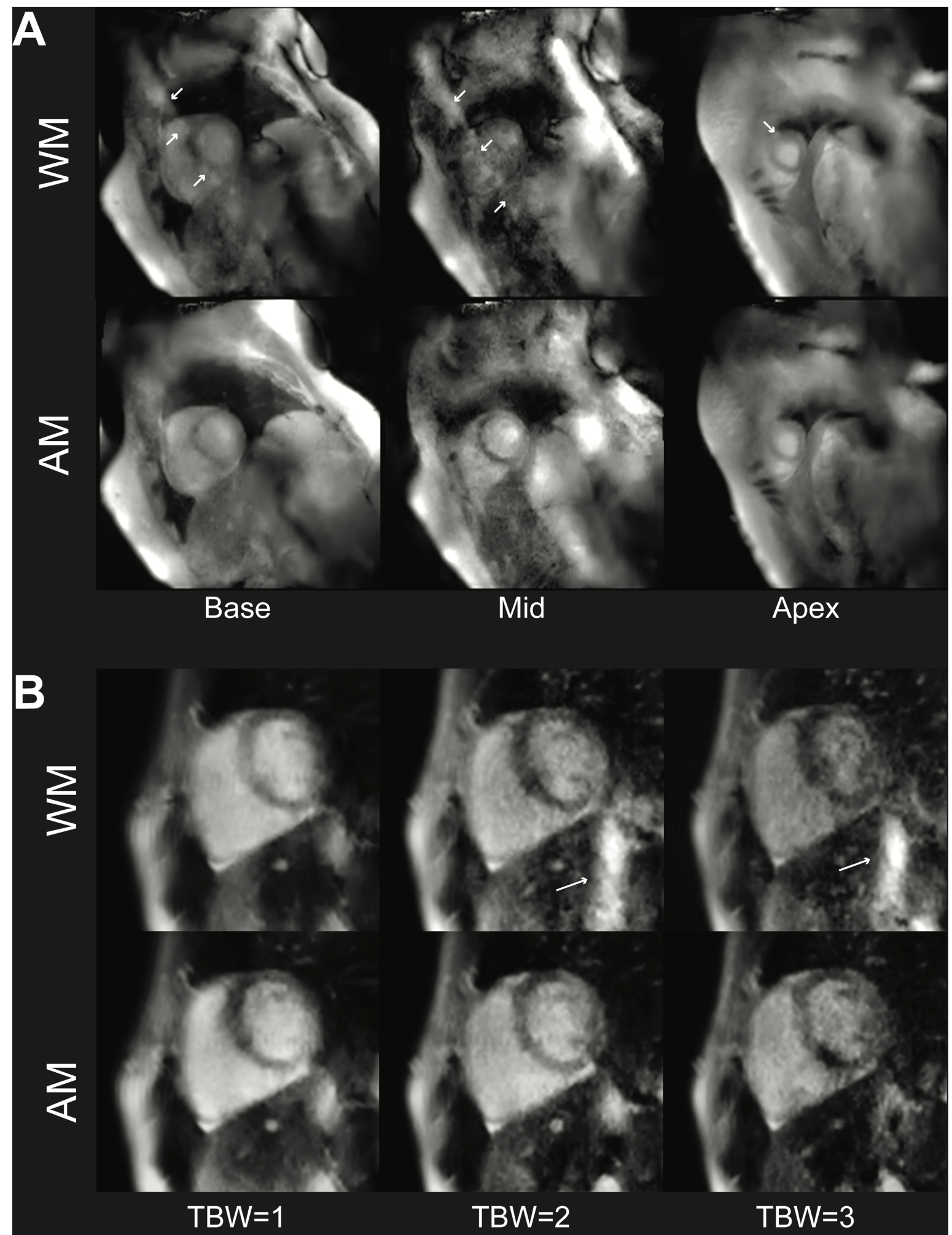

Fig. 5. Comparison between WM and AM for SMS3 Blipped-CAIPIRINHA bSSFP. (A) comparison over the three slices base, mid and apex. (B) TBW value effect on images with WM and AM optimization over the basal slice. The white arrows show the signal leakage artefacts when using WM that completely disappear with AM. WM, Wong modulation; AM, amplitude modulation; TBW; product Time-Band Width. 


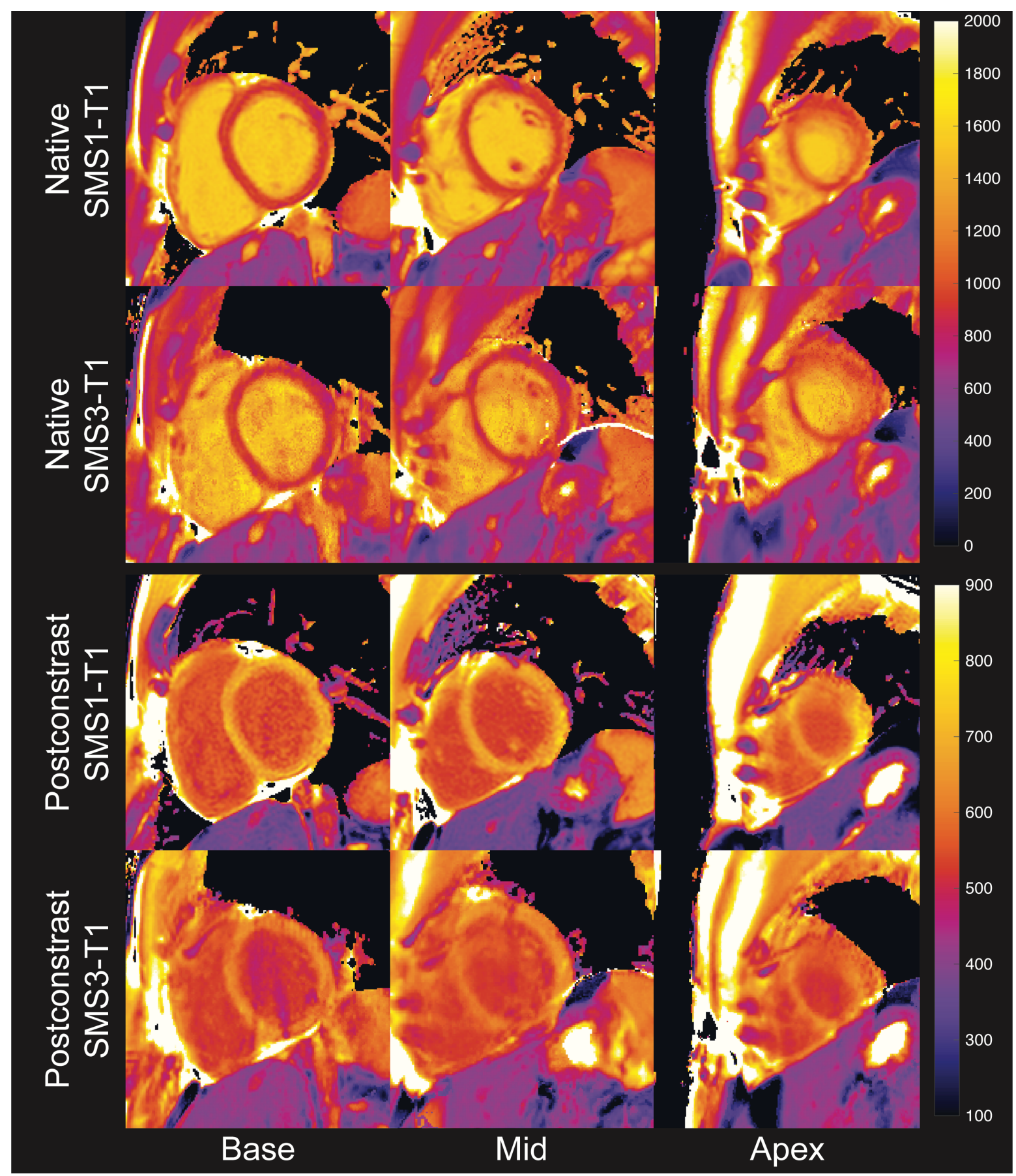

Fig. 6. T1 maps over base, mid and apex using blipped-CAIPIRINHA SMS-MOLLI. From top to bottom: native SMS1-T1 maps using conventional MOLLI, native AM-SMS3-T1 maps using SMSMOLLI, postcontrast (15 min) SMS1-T1 maps and postcontrast (15 min) AM-SMS3-T1. AM, amplitude modulation. 


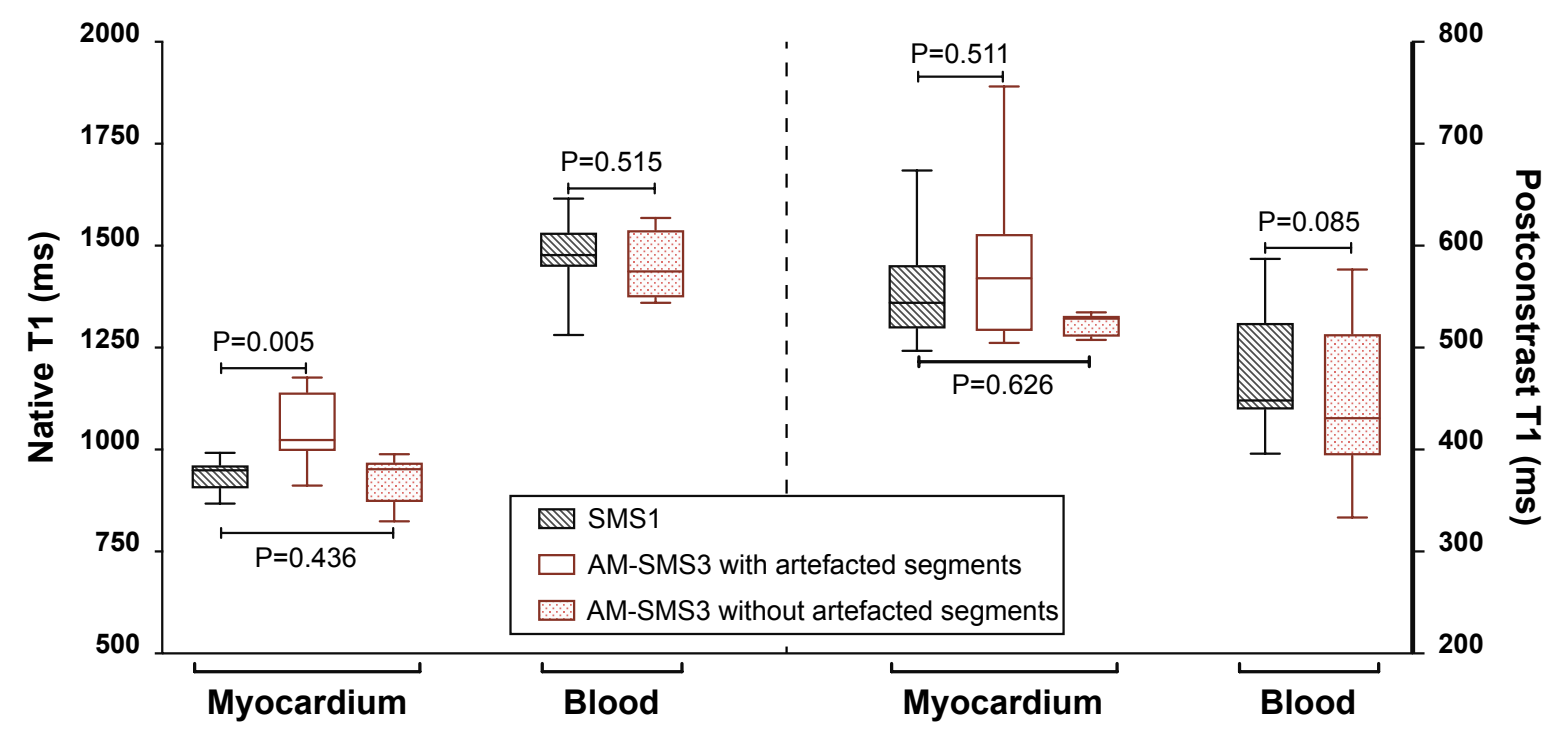

Fig. 7. Box-and-whisker plots of the mean native $\mathrm{T} 1$ and postcontrast $\mathrm{T} 1$ values in the myocardium and the blood using conventional MOLLI and AM-SMS3-MOLLI (with and without the artefact-corrupted LV segments).

\begin{tabular}{|c|c|c|c|c|c|c|c|}
\hline & $\begin{array}{c}\text { SMS1-T1 } \\
\text { (mean } \pm S D)\end{array}$ & $\begin{array}{c}\text { AM-SMS3-T1 } \\
\text { all segments } \\
\text { (mean } \pm S D)\end{array}$ & $\begin{array}{l}\text { Bland- } \\
\text { Altman bias* } \\
\text { all segments } \\
\text { (mean } \pm \mathrm{SD})\end{array}$ & $\begin{array}{l}95 \% \text { limits of } \\
\text { agreement } \\
\text { all segments }\end{array}$ & $\begin{array}{c}\text { AM-SMS3-T1 } \\
\text { no corrupted } \\
\text { segments } \\
\text { (mean } \pm \text { SD) }\end{array}$ & $\begin{array}{l}\begin{array}{c}\text { Bland-Altman } \\
\text { bias* }\end{array} \\
\text { no corrupted } \\
\text { segments } \\
\text { (mean } \pm S D \text { ) }\end{array}$ & $\begin{array}{c}95 \% \text { limits of } \\
\text { agreement } \\
\text { no corrupted } \\
\text { segments }\end{array}$ \\
\hline $\begin{array}{l}\text { Myocardium } \\
\text { native T1 }\end{array}$ & $935.5 \pm 36.1 \mathrm{~ms}$ & $1050.2 \pm 116.2 \mathrm{~ms}$ & $-11.3 \pm 9.8 \%$ & $\begin{array}{c}\text { From }-30.5 \% \\
\text { to } 7.9 \%\end{array}$ & $933.8 \pm 50.2 \mathrm{~ms}$ & $1.5 \% \pm 5.4 \%$ & $\begin{array}{c}\text { From }-9.2 \% \text { to } \\
12.2 \%\end{array}$ \\
\hline $\begin{array}{l}\text { Blood } \\
\text { native T1 }\end{array}$ & $1475.4 \pm 35.9 \mathrm{~ms}$ & $1452.5 \pm 70.3 \mathrm{~ms}$ & $1.4 \pm 7.0 \%$ & $\begin{array}{c}\text { From }-12.3 \% \\
\text { to } 15.1 \%\end{array}$ & NA & NA & NA \\
\hline $\begin{array}{l}\text { Myocardium } \\
\text { postcontrast T1 }\end{array}$ & $556.0 \pm 19.7 \mathrm{~ms}$ & $577.9 \pm 76.3 \mathrm{~ms}$ & $-3.0 \pm 12.5 \%$ & $\begin{array}{c}\text { From }-27.6 \% \\
\text { to } 21.6 \%\end{array}$ & $521.3 \pm 28.1 \mathrm{~ms}$ & $0.7 \% \pm 3.1 \%$ & $\begin{array}{c}\text { From }-5.4 \% \text { to } \\
6.8 \%\end{array}$ \\
\hline $\begin{array}{l}\text { Blood } \\
\text { postcontrast T1 }\end{array}$ & $478 \pm 65.1 \mathrm{~ms}$ & $447.8 \pm 81.5 \mathrm{~ms}$ & $7.1 \pm 10.1 \%$ & $\begin{array}{c}\text { From }-12.7 \% \\
\text { to } 27.0 \%\end{array}$ & NA & NA & NA \\
\hline
\end{tabular}




\section{Appendix A}

In the following figure, the frequency response profile from the bSSFP readout was simulated using Bloch equations for the fat signal and the myocardium signal. The simulation was performed using the following parameters derived from the in vivo experiments and relaxation values at 1.5T from the literature [47], [48]: Fat: $\mathrm{T} 1 / \mathrm{T} 2=250 \mathrm{~ms} / 70 \mathrm{~ms} ;$ myocardium: T1/T2 $=950 \mathrm{~ms} / 55 \mathrm{~ms} ; \mathrm{TE} / \mathrm{TR}=1.37 \mathrm{~ms} / 4.76 \mathrm{~ms}$.

The myocardium response was tested with two flip angles: $35^{\circ}$ (main bands) and $3^{\circ}$ (side-lobes). Fat frequency response was tested only for a flip angle of $3^{\circ}$ to demonstrate that for bSSFP, even with small FA, high signal is observed in positive bands at the frequency offsets corresponding to the conventional dark bands characteristics of bSSFP. Therefore, cardiac SMS-bSSFP displays a unique sensitivity to side-lobe excitations that potentially overlap with epidermal fat in short-axis acquisitions and exhibits positive banding artifacts.

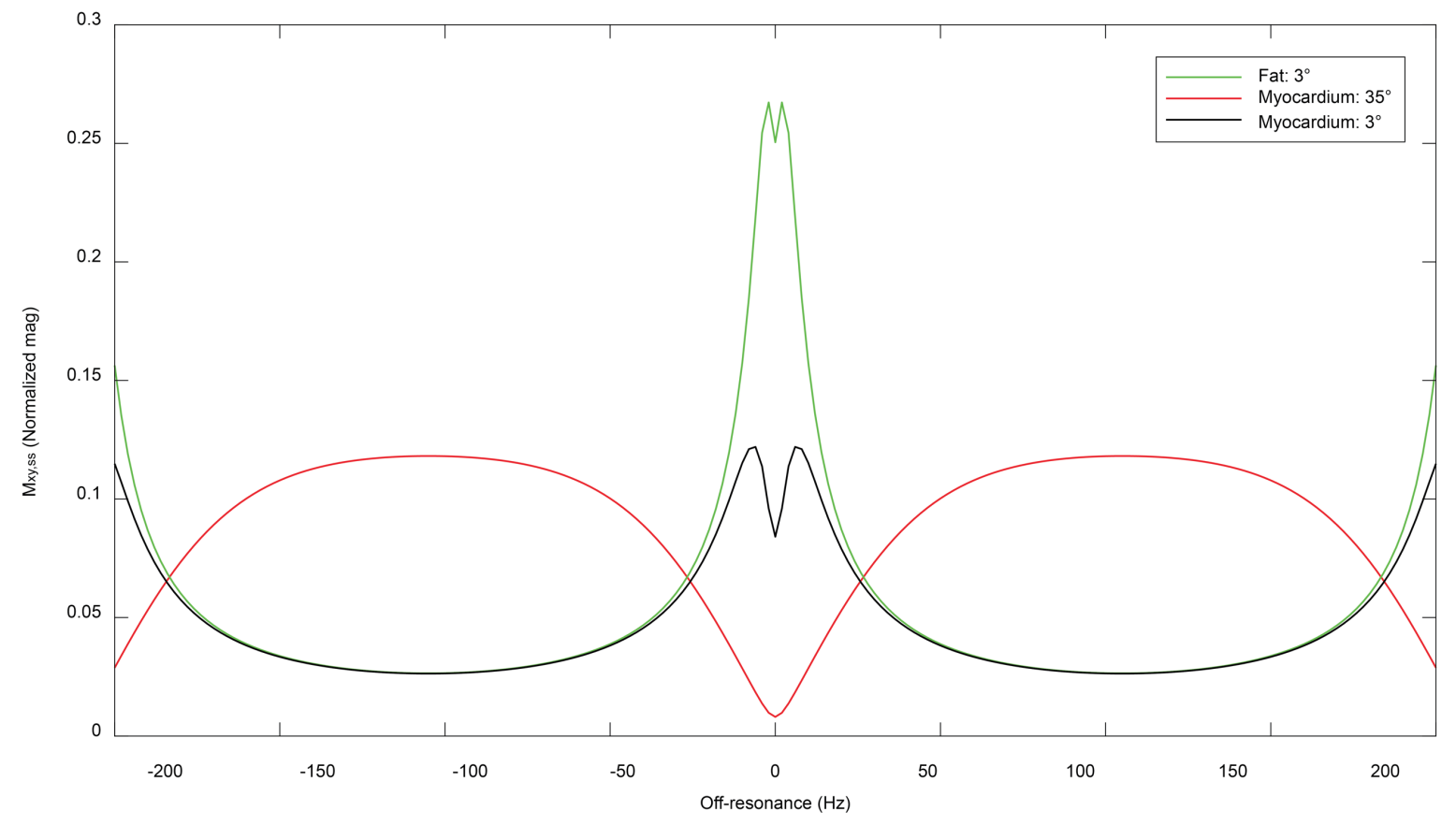




\section{Appendix B}

Table 1. Acquisitions parameters for SNR evaluation on phantom

\begin{tabular}{|c|c|c|c|c|c|c|}
\hline \multirow{2}{*}{ Parameters } & \multicolumn{3}{|c|}{ Slice thickness variation } & \multicolumn{3}{|c|}{ Slice gap variation } \\
\hline & NO-SMS & WM-SMS3 & AM-SMS3 & NO-SMS & WM-SMS3 & AM-SMS3 \\
\hline $\begin{array}{l}\text { Slice } \\
\text { thickness }\end{array}$ & \multicolumn{3}{|c|}{$3,4,5,6,7$ and $8 \mathrm{~mm}$} & \multicolumn{3}{|c|}{$6 \mathrm{~mm}$} \\
\hline Slice gap & \multicolumn{3}{|c|}{$48 \mathrm{~mm}$} & \multicolumn{3}{|c|}{$12,18,24,30,36,42$ and $48 \mathrm{~mm}$} \\
\hline Bandwidth & \multicolumn{6}{|c|}{$600,800,1000,1200$ and $1400 \mathrm{~Hz} / \mathrm{Px}$} \\
\hline TR/TE/FA & \multicolumn{6}{|c|}{$3.81 \mathrm{~ms} / 1.49 \mathrm{~ms} / 30^{\circ}$} \\
\hline FOV & \multicolumn{6}{|c|}{$320 \times 320 \mathrm{~mm}^{2}$} \\
\hline $\begin{array}{l}\text { Spatial } \\
\text { resolution }\end{array}$ & \multicolumn{6}{|c|}{$2 \times 2 \mathrm{~mm}^{2}$} \\
\hline Acceleration & \multicolumn{6}{|c|}{ GRAPPA X2 } \\
\hline
\end{tabular}

Table 2. Acquisition parameters for phantom T1 quantification

\begin{tabular}{|l|c|}
\hline \multicolumn{1}{|c|}{ Parameters } & NO-SMS-T1 \\
\hline Slice thickness & $8 \mathrm{~mm}$ \\
\hline Slice gap & $20 \mathrm{~mm}$ \\
\hline Bandwidth & $800 \mathrm{~Hz} / \mathrm{Px}$ \\
\hline TR/TE/FA & $2.76 \mathrm{~ms} / 1.19 \mathrm{~ms} / 35^{\circ}$ \\
\hline FOV & $300 \times 300 \mathrm{~mm}^{2}$ \\
\hline Spatial resolution & $1.87 \times 1.87 \mathrm{~mm}^{2}$ \\
\hline Time-Bandwidth product & 2 \\
\hline Simulated heart rate & $60 \mathrm{bpm}$ \\
\hline Acceleration & GRAPPA X2 \\
\hline MOLLI scheme & $5(3) 3$ \\
\hline
\end{tabular}

Table 3. Acquisition parameters for WM vs AM on phantom

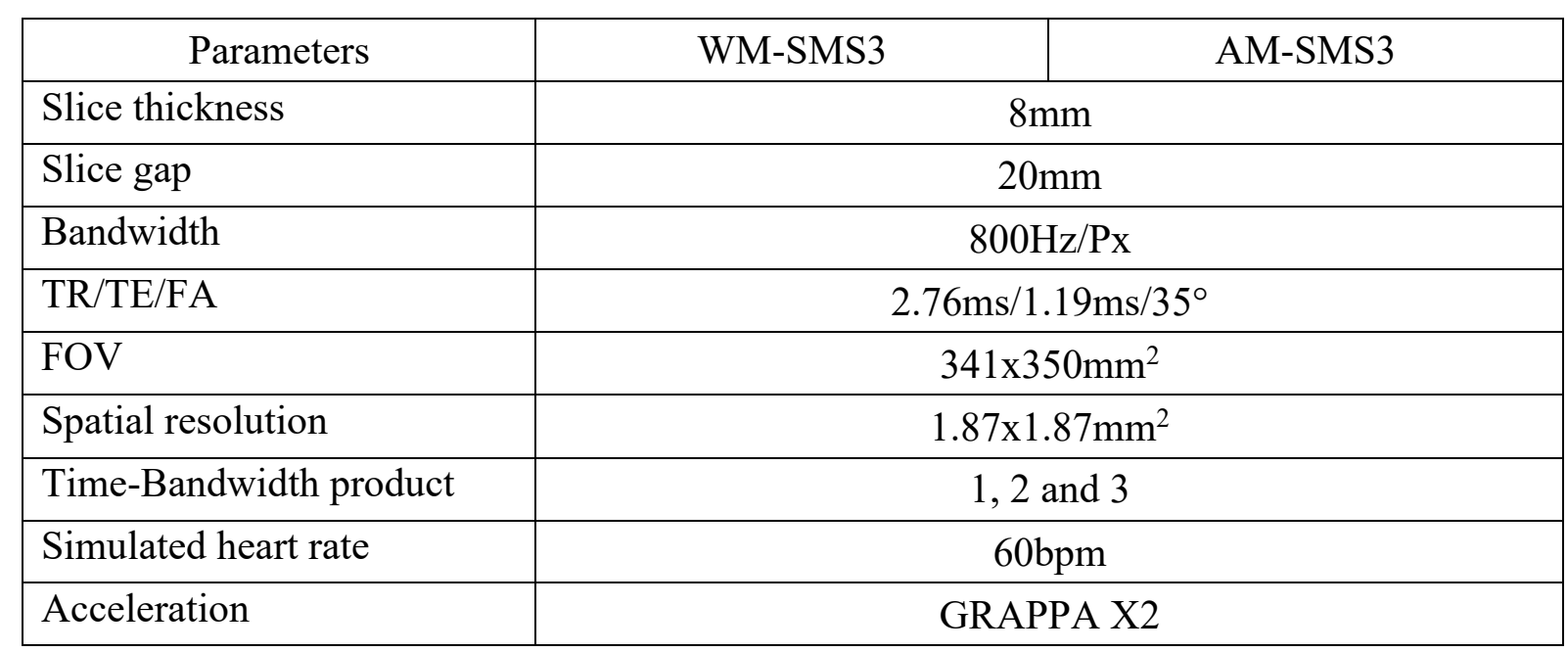


Table 4. In-vivo acquisition parameters for WM vs AM

\begin{tabular}{|c|c|c|}
\hline Parameters & WM-SMS3 & AM-SMS3 \\
\hline Slice thickness & \multicolumn{2}{|c|}{$8 \mathrm{~mm}$} \\
\hline Slice gap & \multicolumn{2}{|c|}{$20 \mathrm{~mm}$} \\
\hline Bandwidth & \multicolumn{2}{|c|}{$800 \mathrm{~Hz} / \mathrm{Px}$} \\
\hline TR/TE/FA & \multicolumn{2}{|c|}{$2.76 \mathrm{~ms} / 1.19 \mathrm{~ms} / 35^{\circ}$} \\
\hline FOV & \multicolumn{2}{|c|}{$341 \times 350 \mathrm{~mm}^{2}$} \\
\hline Spatial resolution & \multicolumn{2}{|c|}{$1.87 \times 1.87 \mathrm{~mm}^{2}$} \\
\hline Time-Bandwidth product & \multicolumn{2}{|c|}{1,2 and 3} \\
\hline Acceleration & \multicolumn{2}{|c|}{ GRAPPA X2 } \\
\hline Breath-hold duration & \multicolumn{2}{|c|}{ Between 11 and $13 \mathrm{~s}$} \\
\hline
\end{tabular}

Table 5. In-vivo acquisition parameters for $\mathrm{T} 1$ quantification

\begin{tabular}{|c|c|c|}
\hline Parameters & NO-SMS-T1 & SMS3-T1 \\
\hline Slice thickness & \multicolumn{2}{|c|}{$8 \mathrm{~mm}$} \\
\hline Slice gap & \multicolumn{2}{|c|}{$20 \mathrm{~mm}$} \\
\hline Bandwidth & \multicolumn{2}{|c|}{$800 \mathrm{~Hz} / \mathrm{Px}$} \\
\hline TR/TE/FA & \multicolumn{2}{|c|}{$2.76 \mathrm{~ms} / 1.19 \mathrm{~ms} / 35^{\circ}$} \\
\hline FOV & \multicolumn{2}{|c|}{$341 \times 350 \mathrm{~mm}^{2}$} \\
\hline Spatial resolution & \multicolumn{2}{|c|}{$1.87 \times 1.87 \mathrm{~mm}^{2}$} \\
\hline Time-Bandwidth product & \multicolumn{2}{|c|}{2} \\
\hline Modulation & NA & WM/AM \\
\hline Acceleration & \multicolumn{2}{|c|}{ GRAPPA X2 } \\
\hline MOLLI scheme & \multicolumn{2}{|c|}{ Native T1: 5(3)3 / postcontrast: 4(1)3(1)2 } \\
\hline Breath-hold duration & \multicolumn{2}{|c|}{ Between 11 and $13 \mathrm{~s}$} \\
\hline
\end{tabular}




\section{Appendix C}
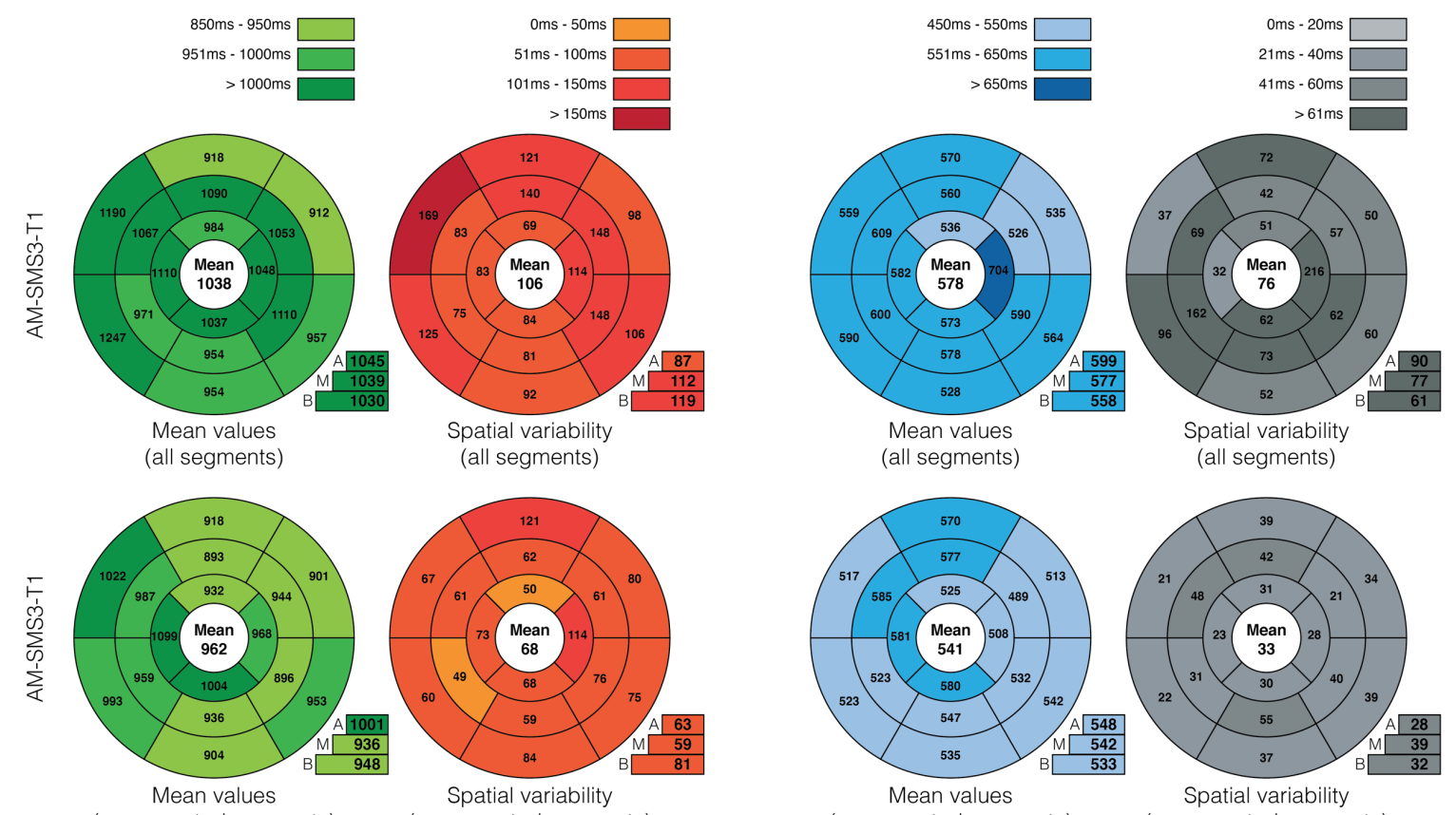

(no corrupted segments) (no corrupted segments) (no corrupted segments)
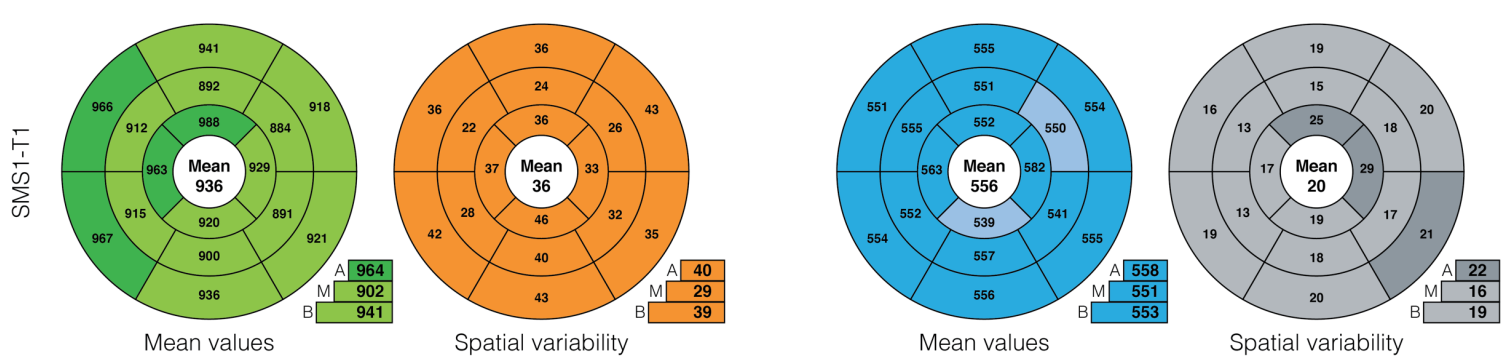

\section{Postcontrast T1}

Mean and spatial variability of T1 values over the 10 subjects for each segment. Native SMS1-T1, post injection SMS1-T1, native AM-SMS3-T1 with and without corrupted segments, and post injection AMSMS3-T1 also with and without corrupted segments are included. 